\title{
Hits per Trial: Basic Analysis of Binomial Data
}

Corwin L. Atwood

Published September 1994

\section{Idaho National Engineering Laboratory \\ EG\&G Idaho, Inc. \\ Idaho Falls, Idaho $\mathbf{8 3 4 1 5}$}

Prepared for the

Reliability and Risk Analysis Branch

Safety Programs Division

Office for Analysis and Evaluation of Operational Data

U.S. Nuclear Regulatory Commission

Washington, DC 20555

Under DOE Idaho Operations Office

Contract DE-AC07-761D01570

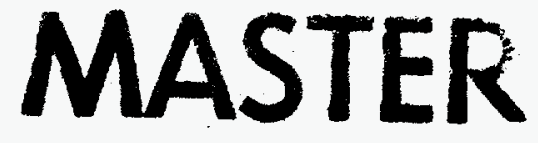

FIN E8205 



\section{DISCLAIMER}

This report was prepared as an account of work sponsored by an agency of the United States Government. Neither the United States Government nor any agency thereof, nor any of their employees, make any warranty, express or implied, or assumes any legal liability or responsibility for the accuracy, completeness, or usefulness of any information, apparatus, product, or process disclosed, or represents that its use would not infringe privately owned rights. Reference herein to any specific commercial product, process, or service by trade name, trademark, manufacturer, or otherwise does not necessarily constitute or imply its endorsement, recommendation, or favoring by the United States Government or any agency thereof. The views and opinions of authors expressed herein do not necessarily state or reflect those of the United States Government or any agency thereof. 


\section{DISCLAIMER}

Portions of this document may be illegible in electronic image products. Images are produced from the best available original document. 


\section{ABSTRACT}

This report presents simple statistical methods for analyzing binomial data, such as the number of failures in some number of demands. It gives point estimates, confidence intervals, and Bayesian intervals for the failure probability. It shows how to compare subsets of the data, both graphically and by statistical tests, and how to look for trends in time. It presents a compound model when the failure probability varies randomly. Examples and SAS programs are given. 


\section{SUMMARY}

This report presents simple statistical methods for analyzing count data of the form $k$ "hits" (events of some type) in $n$ "trials." A common example is $k$ failures on $n$ demands.

First, the simplest case: Consider $n$ independent trials with the probability of a hit on any trial being some number $p$. Formulas are given for the point estimate (maximum likelihood estimate) and confidence interval for $p$, and the Bayes posterior distribution is given, typically based on a noninformative prior distribution.

The assumption of a single $p$ for all the trials can be investigated in several ways. When there are natural subsets of the data, graphical methods and statistical tests are given to investigate whether $p$ is the same for all the subsets. The graphical methods use confidence intervals plotted side by side, and the tests use contingency tables with one row for the hits and one row for the misses. When the individual trials can be arranged sequentially (for example, in chronological order), a cumulative plot of the hits helps show whether $p$ is constant throughout the sequence. Further, the Wilcoxon-Mann-Whitney test can test this assumption against the alternative that $p$ is largest at one end of the sequence and smallest at the other end.

The other assumption of the simple model is that the trials are statistically independent. Investigating this assumption may only require careful thinking, not statistical analysis. However, the runs test can be used to test for serial dependence.

A compound model is presented for the case when the data come from a number of distinct sources and $p$ varies randomly among the sources. Here $p$ is assumed to follow a beta distribution, whose parameters are estimated from the data. The value of $p_{i}$ for the $i$ th data source is then estimated by the empirical Bayes method.

Every method is illustrated by an example. SAS programs are given for all the calculation procedures. 


\section{FOREWORD}

The Office for Analysis and Evaluation of Operational Data (AEOD) of the U.S. Nuclear Regulatory Commission (NRC) is continually reviewing data from U.S. commercial nuclear power plants. Statistical analysis forms an important part of this work. Accordingly, the Reliability and Risk Analysis Branch of AEOD has asked the Idaho National Engineering Laboratory (INEL) to write a series of reports presenting the appropriate statistical tools for the kinds of data most commonly encountered. These reports are being written in parallel with a series of studies on the performance of safety systems in nuclear power plants, and reflect the influence of those studies.

The reports are expected to provide tools and guidance to analysts of NRC data, although the statistical methods can, by their nature, be applied to data from many other fields. The reports are intended to quickly help a new user. For example, the reports on statistical methods should be readable and immediately usable by a person with training in statistics but with no experience analyzing such data. The report on collecting operational data should be understandable by anyone with a technical background, although the examples are slanted towards an engineer with nuclear experience. In addition, the reports should be usable as texts or references in short courses for persons with less training.

The first three reports in this series, appearing in September, 1994, are

Collecting Operational Event Data for Statistical Analysis, EGG-RAAM-11086, by Corwin L. Atwood

Hits per Trial: Basic Analysis of Binomial Data, EGG-RAAM-11041, by Corwin L. Atwood

Events in Time: Basic Analysis of Poisson Data, EGG-RAAM-11088, by M. E. Engelhardt

Other reports in the series are planned for 1995. 


\section{ACKNOWLEDGMENTS}

Mark DeHaan, Cindy Gentillon and I collaborated in developing the SAS programs. Dana Kelly carefully reviewed an early draft, Max Engelhardt gave useful advice on several technical issues, and the probing comments of Cindy Gentillon helped me to clarify the presentation and convinced me to add several sections. Harry Martz suggested the KassSteffey improvement to empirical Bayes intervals, and helped improve the overall presentation. 


\section{CONTENTS}

ABSTRACT $\ldots \ldots \ldots \ldots \ldots \ldots \ldots \ldots \ldots \ldots \ldots \ldots \ldots \ldots \ldots \ldots$

SUMMARY $\ldots \ldots \ldots \ldots \ldots \ldots \ldots \ldots \ldots \ldots \ldots \ldots \ldots \ldots \ldots \ldots \ldots \ldots$

FOREWORD $\ldots \ldots \ldots \ldots \ldots \ldots \ldots \ldots \ldots \ldots \ldots \ldots \ldots \ldots \ldots$

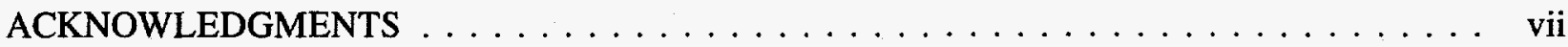

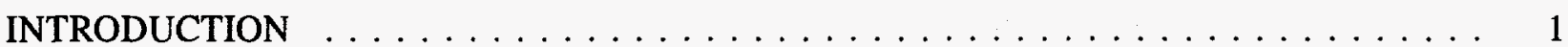

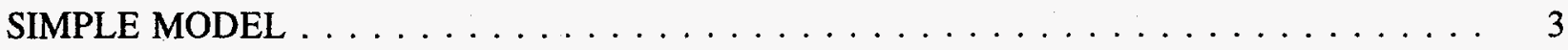

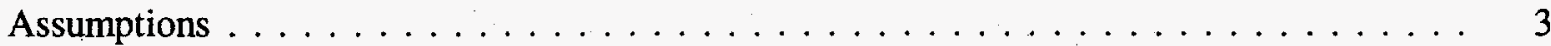

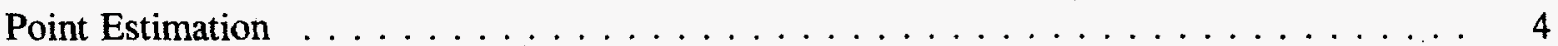

Standard Deviation of Estimator $\ldots \ldots \ldots \ldots \ldots \ldots \ldots \ldots \ldots \ldots \ldots \ldots$

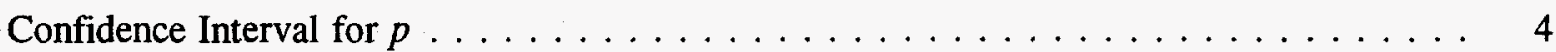

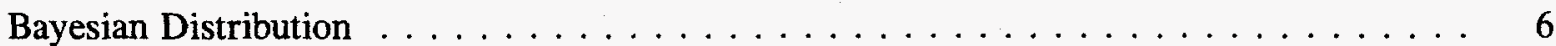

CHECKING THE MODEL ASSUMPTIONS $\ldots \ldots \ldots \ldots \ldots \ldots \ldots \ldots \ldots$

Assumption of Same $p$ in Different Subsets . . . . . . . . . . . . . . . . 10

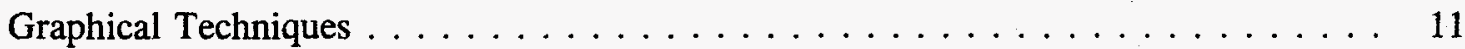

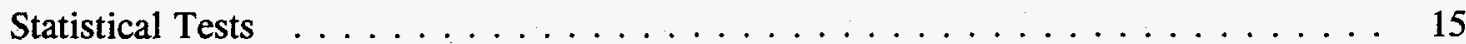

Assumption of Constant $p$ for Trials in Sequence $\ldots \ldots \ldots \ldots \ldots \ldots \ldots$

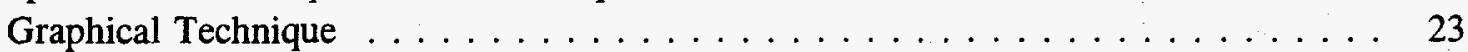

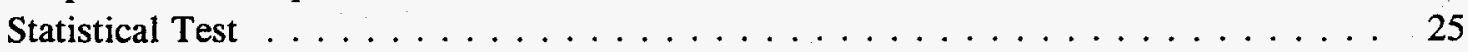

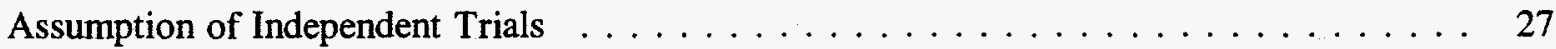

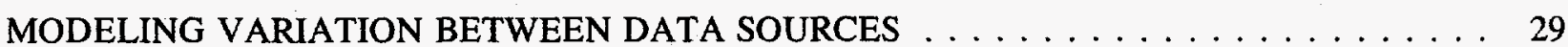

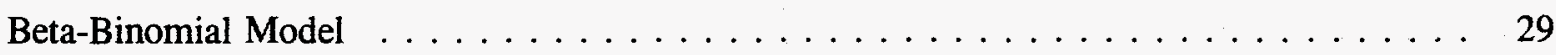

Empirical Bayes Estimates $\ldots \ldots \ldots \ldots \ldots \ldots \ldots$

REVIEW AND GUIDELINES $\ldots \ldots \ldots \ldots \ldots \ldots \ldots \ldots \ldots \ldots \ldots \ldots$

The Simple Model and Its Data Analysis $\ldots \ldots \ldots \ldots \ldots \ldots \ldots \ldots$

Checking the Assumptions of the Simple Model $\ldots \ldots \ldots \ldots \ldots \ldots \ldots$

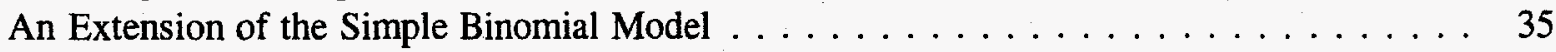

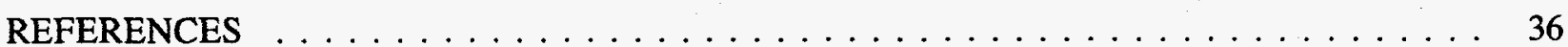

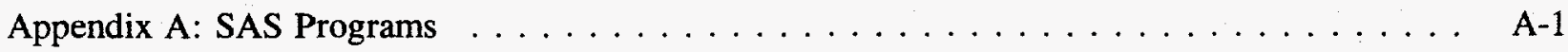

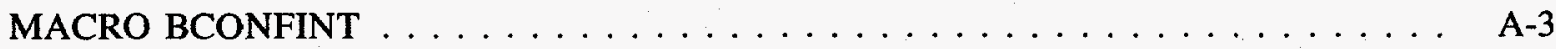

MACRO BBAYES $\ldots \ldots \ldots \ldots \ldots \ldots \ldots \ldots \ldots \ldots \ldots \ldots \ldots \ldots$

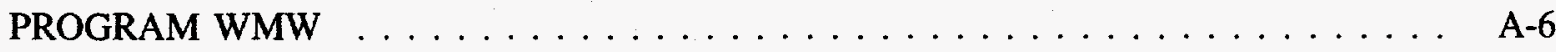




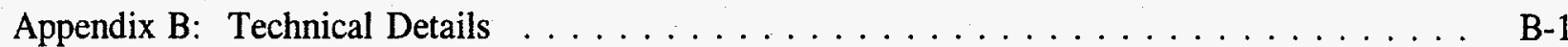

CONFIDENCE INTERVALS AND BAYES INTERVALS $\ldots \ldots \ldots \ldots \ldots \ldots$ B-3

Confidence Intervals $\ldots \ldots \ldots \ldots \ldots \ldots \ldots \ldots \ldots \ldots \ldots$ B-3

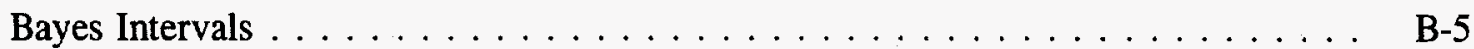

EMPIRICAL BAYES METHOD $\ldots \ldots \ldots \ldots \ldots \ldots \ldots \ldots \ldots \ldots \ldots$ B-7

Beta-Binomial Likelihood $\ldots \ldots \ldots \ldots \ldots \ldots \ldots \ldots \ldots \ldots \ldots \ldots \ldots$ B-7

Maximum Likelihood Estimation . . . . . . . . . . . . . . . B-7

Adjustment for Uncertainty in Maximum Likelihood Estimates . . . . . . . . . . B B-9

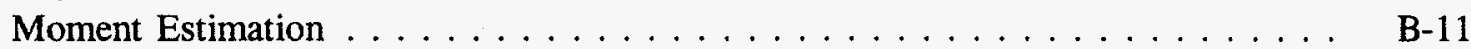

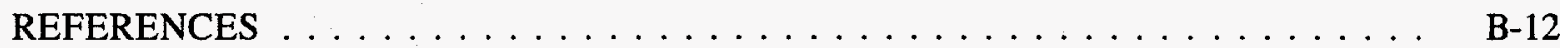

\section{FIGURES}

Figure 1. Jeffreys noninformative prior distribution for binomial model. $\ldots \ldots \ldots \ldots \ldots 7$

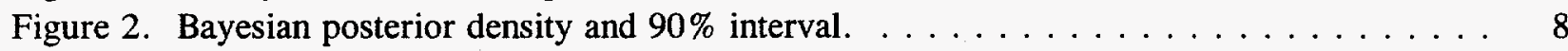

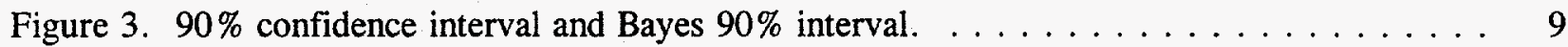

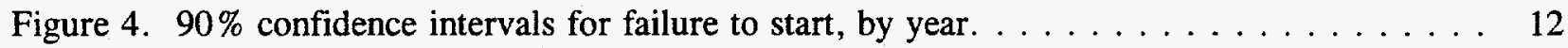

Figure 5. Failures to start $1987-92$, by plant, ordered alphabetically $\ldots \ldots \ldots \ldots \ldots \ldots$

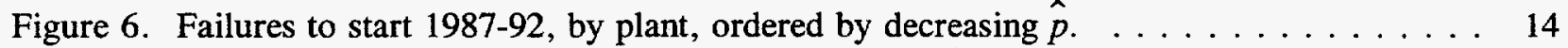

Figure 7. Cumulative count of losses of safety function, among all reported events. . . . . . 24

Figure 8. Bayes means and $90 \%$ intervals for $p_{i}$, with each plant estimate based on a

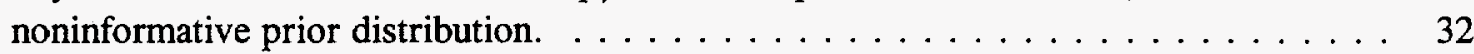

Figure 9. Empirical Bayes means and $90 \%$ intervals for $p_{i}$ by plant $\ldots \ldots \ldots \ldots \ldots \ldots$

\section{TABLES}

Table 1. Contingency table using failures to start on demand, by year. . . . . . . . . . . 15

Table 2. Analysis of failures to start on demand, by year. $\ldots \ldots \ldots \ldots \ldots$

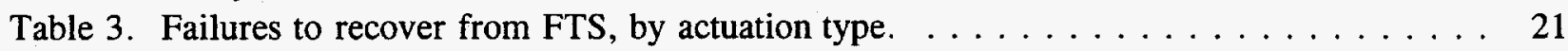

Table 4. Wilcoxon-Mann-Whitney test of constant hit probability in Licensee Event Reports. . . 26

\section{EXAMPLES}

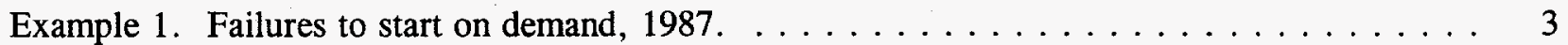

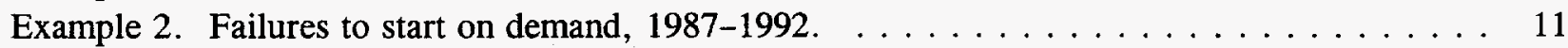

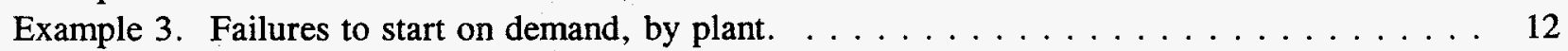

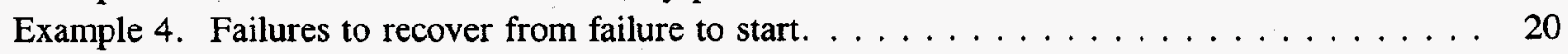

Example 5. Licensee Event Reports with safety function lost or available. . . . . . . . . . 23 
Example 5. Licensee Event Reports with safety function lost or available. 


\section{Hits per Trial: \\ Basic Analysis of Binomial Data}

\section{INTRODUCTION}

This report presents simple statistical methods for analyzing count data of a particular form: there is a finite number, $n$, of "trials," and each trial can be a "hit" or a "miss." Only basic methods are given here, with more advanced methods deferred to a later report.

Here are some examples of the type of data considered. The first example is the classic example in probability courses, while the other examples arise in the analysis of failure data.

- Toss a coin $n$ times and count the number of heads. There are $n$ trials, and each head is a hit.

- A standby component or system is demanded $n$ times; a hit occurs whenever the component or system fails to operate on demand.

- There were $n$ failures observed, and a hit occurs whenever the cause of a failure was personnel error.

- There were $n$ events reported in Licensee Event Reports (LERs), and a hit occurs whenever the event was a true failure, that is, the system safety function was lost.

Note the common pattern of all these examples: there were $n$ events of some type, and a hit is counted whenever an event satisfies some condition. The second example, the number of failures in $n$ demands, is the most common example in the context of failure data, but the others are mentioned to suggest the breadth of situations that have the same mathematical form.

This report first treats the simple model, with a single parameter to be estimated. Then, it gives ways to check the correctness of the simple model assumptions for data sets. It presents a compound model for situations where subsets of the data have different probabilities of a hit. A concluding section reviews the methods and gives guidelines for their use. Every definition and procedure is illustrated with 
an example, and references are given for all but the most basic formulas. Appendix A lists SAS (1988) $^{2}$ programs to do all of the examples. Appendix B treats some topics in more technical detail.

The analysis methods of this report are illustrated by data from a 1993 draft study. That study considered failures of the high pressure coolant injection (HPCI) system in commercial nuclear power plants of the boiling water reactor design. HPCI is a standby system, demanded only on conditions of low water level in the reactor vessel (which automatically initiate unplanned actuations of the system) and during periodic surveillance tests. Any failure of the system to meet the technical specifications, including any failure to operate during unplanned actuations or surveillance tests, must be reported in a Licensee Event Report. These reports were read, and the resulting data formed the basis of the draft study. After peer review, some of the events in the data set were reclassified. Therefore, the example data sets in this report are not real, but they are like real data sets. They are given as illustrations only, and no inferences should be drawn from the numerical conclusions.

a. Mention of specific products and/or manufacturers in this document implies neither endorsement or preference, nor disapproval by the U.S. Government, any of its agencies, or EG\&G Idaho, Inc. of the use of a specific product for any purpose. 


\section{SIMPLE MODEL}

The simple model is illustrated by the following example.

Example 1. Failures to start on demand, 1987.

In one year of data covering all commercial U.S. boiling water reactors with a high pressure coolant injection (HPCI) system, there were 30 demands on the system, and the system failed to start 3 times.

\section{Assumptions}

The usual assumptions for the simple model are:

1. Each trial is a hit with some probability $p$, and is a miss with probability $1-p$.

2. Occurrences of hits for different trials are statistically independent; that is, the probability of a hit on one trial is not affected by what happens on other trials.

3. The data consist of the count of hits in a known number of trials. The number of trials is considered fixed, and the number of hits is random.

Under these assumptions, the number of hits in $n$ trials has a binomial $(n, p)$ distribution. Statistics books give information about the binomial distribution; the next subsections of this report give the facts needed for data analysis.

In Example 1, a trial is a demand on the system, and a hit is failure of the system to start. The number of trials is $n=30$ and the number of hits is $k=3$. An important aspect of Assumption 1 is that the probability of failure is the same on every trial, even though the trials occurred at different plants over the course of a year. Assumption 2 says that the occurrence of failure or success on one trial does not affect the probability of failure or success on other trials. In the real world, probably neither of these assumptions is perfectly true, but for now we assume the simple model and perform the simple analysis. Assumption 3 says that the number of demands on the system is considered fixed while the outcome, success or failure, is considered random. This is reasonable for this example, although there are other situations, such as product testing, when the process can be terminated after a fixed number of failures, 
and therefore a random number of trials. Such a case would be a geometric model, not a binomial model.

\section{Point Estimation}

Suppose $k$ hits occur in $n$ trials. The hit probability $p$ is estimated by $\hat{p}=k / n$. This is the maximum likelihood estimate (MLE), defined in statistics books. In Example 1, $p$, the probability of failure on demand, is estimated by $\hat{p}=3 / 30=0.10$.

\section{Standard Deviation of Estimator}

The number of hits is random. One number was observed, but if the trials were repeated, a different number of hits might be observed. Therefore, the estimator is random, and our estimate is the value it happened to take this time. The standard deviation of the estimator is $[p(1-p) / n]^{1 / 2}$. Substitute $\hat{p}$ for $p$, yielding an estimate of the standard deviation. In Example 1, this estimate is

$[0.10 \times(1-0.10) / 30]^{1 / 2}=0.05477$

\section{Confidence Interval for $p$}

The following material is drawn from Johnson and Kotz (1969, Section 3.7.2). A confidence interval for $p$ can be shown from first principles to be expressible in terms of quantiles of a beta distribution. The confidence limits are then commonly reexpressed in terms of quantiles of the $F$ distribution. The beta formulation is presented here because it allows a direct natural comparison between confidence intervals and Bayes intervals. The formulation with an $F$ distribution is presented because it allows a user to calculate confidence intervals from published tables. Impatient readers may skip the formulas, and curious readers can find more details in Appendix B.

First, the fundamental facts about the beta distribution are summarized. A beta( $\alpha, \beta)$ random variable $X$ has density proportional to $x^{\alpha-1}(1-x)^{\beta-1}$ for $0 \leq x \leq 1$. The parameters of the distribution, $\alpha$ and $\beta$, must both be positive. The mean and variance of the distribution are

$\mu=\alpha /(\alpha+\beta)$

$\sigma^{2}=\mu(1-\mu) /(\alpha+\beta+1)$.

When $X$ has a beta $(\alpha, \beta)$ distribution, define the notation 
$I(c ; \alpha, \beta)=P(X \leq c)$.

$I(c ; \alpha, \beta)$ is the integral of the beta density from 0 to $c$.

Here is the relation to confidence intervals for $p$. As before, let $k$ denote the number of hits in $n$ trials. It can be shown from first principles (see Appendix B) that the lower and upper confidence limits on $p$, denoted $p_{\text {conf }, \mathrm{L}}$ and $p_{\mathrm{conf}, \mathrm{U}}$, satisfy

$I\left(p_{\text {conf,L }} ; k, n-k+1\right)=\alpha / 2$

$I\left(p_{\text {conf,U }} ; k+1, n-k\right)=1-\alpha / 2$

Some people prefer an alternative reexpression in terms of the $F$ distribution, as explained in Appendix B and summarized here. The fundamental relation is that the value $p$ satisfying

$I\left(p ; k_{1}, k_{2}\right)=c$

is

$p=2 k_{1} F_{\mathrm{c}} /\left(2 k_{2}+2 k_{1} F_{\mathrm{c}}\right)$

where $F_{\mathrm{c}}$ is the $c$ quantile of the $F$ distribution with $2 k_{1}$ and $2 k_{2}$ degrees of freedom. Therefore, Equations (2a) and (2b) can be translated: The lower limit is

$p_{\text {conf }, \mathrm{L}}=\nu_{1} F_{\alpha / 2} /\left(\nu_{2}+\nu_{1} F_{\alpha / 2}\right)$

where

$\nu_{1}=2 k$

$\nu_{2}=2(n-k+1)$

$F_{\alpha / 2}=$ the $\alpha / 2$ quantile of the $F$ distribution with $\nu_{1}$ and $\nu_{2}$ degrees of freedom.

If $k=0$, then $\nu_{1}$ equals 0 and the $F$ distribution is degenerate, concentrated at 0 ; in that case, simply set $p_{\text {conf, } \mathrm{L}}=0$. Similarly, the upper limit is

$p_{\text {conf, } \mathrm{U}}=\nu_{1} F_{1-\alpha / 2} /\left(\nu_{2}+\nu_{1} F_{1-\alpha / 2}\right)$ 
where

$\nu_{1} \quad=2(k+1)$

$\nu_{2} \quad=2(n-k)$

$F_{1-\alpha / 2}=$ the $(1-\alpha / 2)$ quantile of the $F$ distribution with $\nu_{1}$ and $\nu_{2}$ degrees of freedom.

If $k=n$, then $\nu_{2}$ equals 0 and the $F$ distribution is not defined; in that case, set $p_{\text {conf,U }}=1$.

In Example 1, suppose a $90 \%$ interval is to be found; then $\alpha=0.10$, so that $1-\alpha / 2=0.95$. For the lower limit, $\nu_{1}$ equals 6 and $\nu_{2}$ equals 56 , and $F_{\alpha / 2}$ is the 0.05 point of the $F$ distribution with 6 and 56 degrees of freedom. This is easy to find with a computer package, and often possible with published tables. Those who use published tables will need extensive tables, such as those in Martz and Waller (1991), and knowledge of the relation $F_{\alpha / 2}\left(\nu_{1}, \nu_{2}\right)=1 / F_{1-\alpha / 2}\left(\nu_{2}, \nu_{1}\right)$. The package SAS finds $F_{0,05}$ to be 0.26704 , so that

$p_{\text {conf }, \mathrm{L}}=6 \times 0.26704 /(56+6 \times 0.26704)=0.0278$.

For the upper limit, $\nu_{1}$ equals 8 and $\nu_{2}$ equals 54 , and $F_{1-\alpha / 2}$ is the 0.95 point of the $F$ distribution with 8 and 54 degrees of freedom, which SAS computes to be 2.1152. Therefore $p_{\text {conf, }}$ is found by SAS to equal 0.2386 , and the $90 \%$ confidence interval is

$(0.028,0.239)$.

The SAS program bconfint, given in Appendix A, calculated the interval. In summary, a $90 \%$ confidence interval for $p$ is $(0.028,0.239)$. Older statistics books mention an approximate confidence interval, based on the approximate normality of $\hat{p}$. In the modern computing environment, there is no reason for ever using that interval in a printed report.

\section{Bayesian Distribution}

If $p$ is to be input to a probabilistic risk assessment (PRA) code such as IRRAS (Russell et al. 1992, 1993), it must be given a distribution, often called an "uncertainty distribution" by PRA workers. The standard Bayesian method is as follows. Assume a prior distribution for $p$, and let $k$ be the observed number of hits and $n-k$ the observed number of misses. By far the most convenient form for the prior is a beta $\left(\alpha_{\text {prior }}, \beta_{\text {prior }}\right)$ distribution. This is convenient because updating the prior distribution with the data produces a posterior distribution of the form $\operatorname{beta}\left(\alpha_{\text {post }}, \beta_{\text {post }}\right)$, with $\alpha_{\text {post }}=\alpha_{\text {prior }}+k$ and $\beta_{\text {post }}=\beta_{\text {prior }}+$ 
$(n-k)$. This relation of the beta to the binomial distribution is presented in many statistics books that treat Bayesian methods.

The prior distribution can reflect knowledge about $p$ for the system or component being studied, acquired from sources other than the data now being analyzed. Ways to quantify such knowledge are beyond the scope of this report. A concentrated distribution (small variance, large value of $\alpha_{\text {prior }}+\beta_{\text {prior }}$ ) represents much presumed prior knowledge. A diffuse prior (large variance, small value of $\alpha_{\text {prior }}+\beta_{\text {prior }}$ ) represents very little prior knowledge of $p$.

In particular, the Jeffreys "noninformative prior" is beta( $1 / 2,1 / 2)$; see Box and Tiao (1973), Sections 1.3.4-1.3.5. This distribution is shown in Figure 1. It is not the uniform distribution, which is a beta(1, 1) distribution, but instead rises sharply at the two ends of the interval $(0,1)$. Although the uniform distribution is sometimes used to model no prior information, there are theoretical reasons for preferring the Jeffreys noninformative prior. These reasons are given by Box and Tiao, and are suggested by the comparison with confidence intervals presented below and in Appendix B.

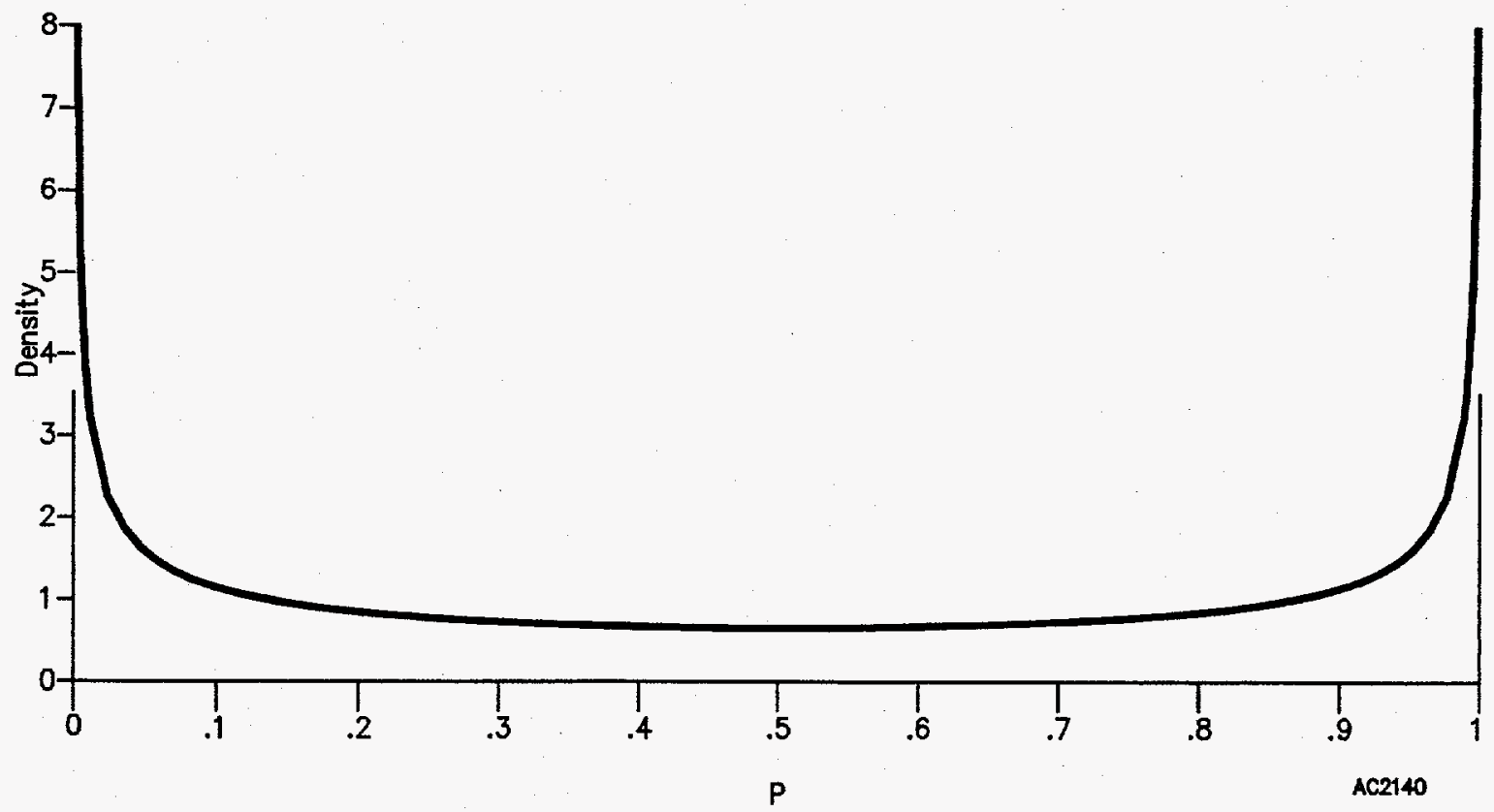

Figure 1. Jeffreys noninformative prior distribution for binomial model.

The Bayes posterior distribution for $p$, based on the Jeffreys noninformative prior, is beta $(k+1 / 2$, $n-k+1 / 2$ ). The mean of the distribution (one of the required inputs to IRRAS) is $(k+1 / 2) /(n+1)$. 
The endpoints of an interval with content $1-\alpha$ are the $\alpha / 2$ and $(1-\alpha / 2)$ quantiles of the posterior beta distribution. That is, the endpoints, denoted $p_{\text {Bayes, } \mathrm{L}}$ and $p_{\text {Bayes, } \mathrm{U}}$, satisfy

$I\left(p_{\text {Bayes }, \mathrm{L}} ; k+1 / 2, n-k+1 / 2\right)=\alpha / 2$

$I\left(p_{\text {Bayes }, \mathrm{U}} ; k+1 / 2, n-k+1 / 2\right)=1-\alpha / 2$

where, as before, $I(c ; \alpha, \beta)$ denotes the probability that a beta $(\alpha, \beta)$ random variable is $\leq c$. Just as for confidence intervals, this could be reexpressed in terms of the $F$ distribution using Equation (3). The distribution and interval are illustrated in Figure 2, for the data of Example 1. Unlike the prior, the posterior density has a single maximum, which is near the observed fraction of hits, 3/30. The density is virtually zero in much of the interval $(0,1)$. Figure 2 also shows the $90 \%$ interval, corresponding to the unshaded region under the density curve.

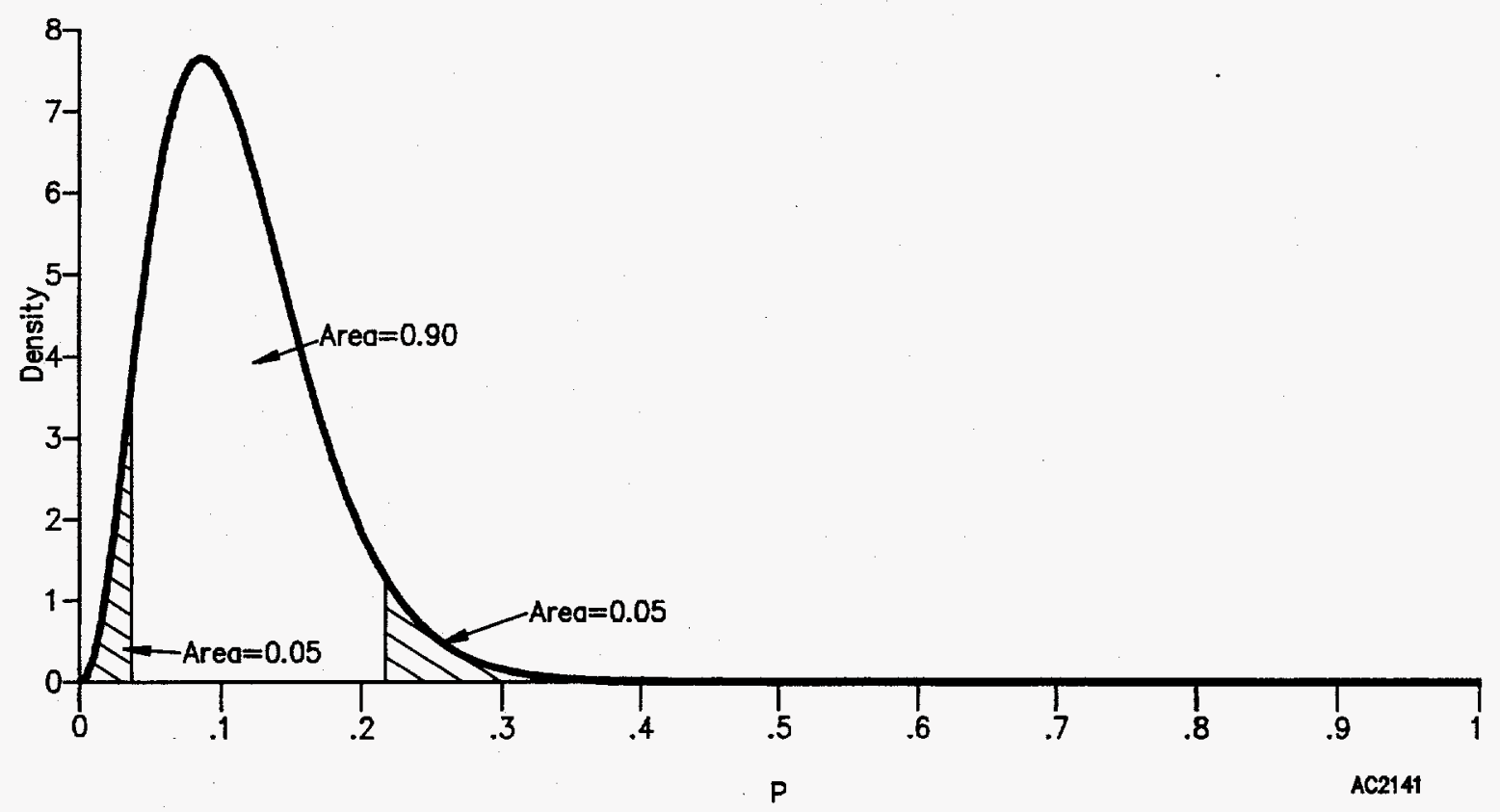

Figure 2. Bayesian posterior density and $90 \%$ interval.

It is very instructive to compare the above equations with Equations (2a) and (2b). The equations are the same, except the parameters in (4a) are the same as in (4b), and are the averages of the parameters in (2a) and (2b). That is, the first parameter, $k+1 / 2$, in Equations (4a) and (4b) is the average of the parameter $k$ in Equation (2a) and $k+1$ in Equation $(2 \mathrm{~b})$, and the second parameters are similarly 
related. This results in a Bayesian interval estimate that is numerically close to the confidence interval, but a little shorter at each end.

In Example 1, the beta distribution based on a noninformative prior distribution is beta $(3.5,27.5)$, with mean $3.5 / 31=0.1129$. The two inputs to IRRAS are the mean 0.1129 and the second parameter 27.5. (See Figure 31 of Russell et al. 1992, or Section 9.3 of Russell et al. 1993.) A 90\% Bayesian interval for $p$ is

$(0.037,0.217)$.

This interval was found by the SAS program bbayes, given in Appendix A. Figure 3 shows a comparison of the $90 \%$ confidence interval and the Bayes $90 \%$ interval. The dots on the intervals show the point estimates, the MLE on the confidence interval and the Bayes posterior mean on the Bayesian interval. The Bayes interval is numerically similar to the confidence interval, but a little shorter, and the Bayes mean is shifted from the MLE toward the prior mean of 0.5 .

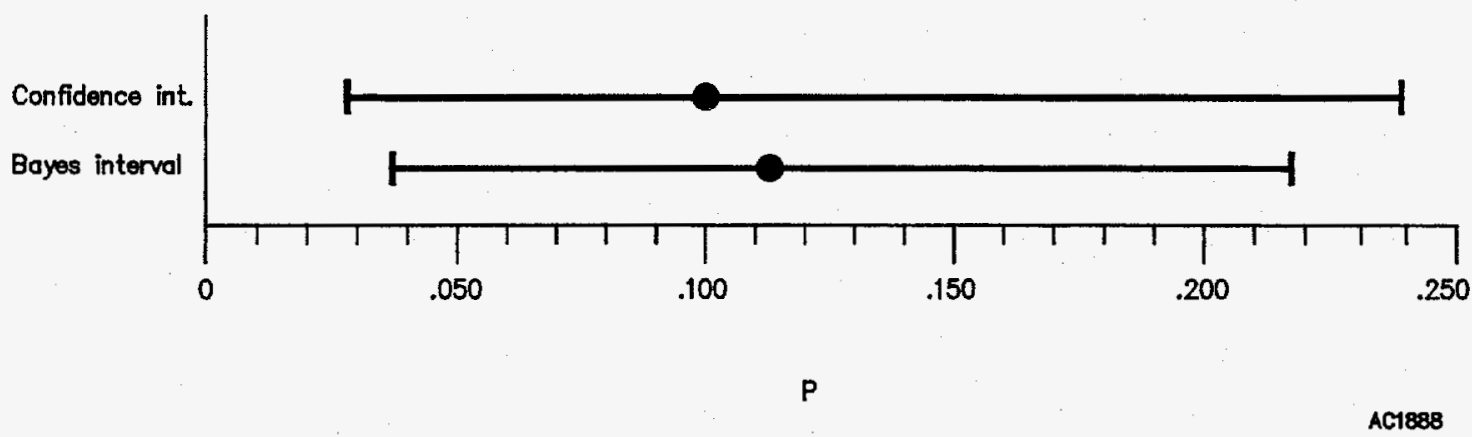

Figure 3. $90 \%$ confidence interval and Bayes $90 \%$ interval.

The similarity of the two intervals would be even closer if the data set were larger-more hits in more trials. For a given number of trials, the two intervals are least similar when there are no hits or no misses: if Example 1 were changed to have 0 hits in 30 trials, the triple (lower bound, point estimate, upper bound) would be $(0,0,0.095)$ for the confidence interval and $(0.0001,0.016,0.062)$ for the Bayesian interval. Appendix B gives more details about the relation of a Bayes interval and a confidence interval. 


\section{CHECKING THE MODEL ASSUMPTIONS}

An important part of any data analysis involves checking the assumptions of the model. Several possible violations of the simple binomial assumptions are considered here. For each case, one or more graphical techniques are given, to help the analyst understand what the data set shows. Then formal statistical tools are presented, which quantify the strength of any evidence against the assumption being considered.

\section{Assumption of Same $p$ in Different Subsets}

Recall that Assumption 1 implied that there is one $p$ for the entire data set. The correctness of this assumption can be investigated by analyzing natural subsets of the data separately, and comparing the $p$ 's for the various subsets. The study team working together can best suggest "natural subsets," better than can a statistician alone or even an engineer alone.

One common way to subdivide the data is by time. For example, make a subset for each year, or each calendar quarter, or some other division of time. Too fine a partition can result in too sparse data, while too coarse a partition can hide some of the differences over time. Another possible way to divide the data by time is to consider data before and after some important event, such as the issuance of a rule or a retrofit of a system.

Other common ways to subdivide the data are by location, function, or hardware design. It is natural to consider different systems separately, but if similar systems, such as HPCI and RCIC, are initially considered together, divide them to see if there are differences in the $p$ 's. The data can also be divided by considering each plant as a separate data generator, or by considering a peer group of similar plants as defining one subset of the data.

The analysts must ask: "Where would I expect to find differences in the p's, if differences exist?" Then subdivide the data accordingly, and carry out the investigations described below to see if the differences are really there.

The techniques below will be illustrated primarily by the following example. Like Example 1, this data set came from a draft study, and the data have since been reviewed and changed. Therefore, no conclusions should be drawn from the numbers. In Example 2, the subsets to be compared are years Note that Example 1 is the year 1987 of Example 2. 
Example 2. Failures to start on demand, 1987-1992.

The HPCI system at U.S. BWRs had the listed numbers of demands and failures to start in the years 1987 through 1992.

\section{Year $\quad \underline{\text { Failures }}$ Demands}

$1987 \quad 3 \quad 30$

$\begin{array}{lll}1988 & 4 & 24\end{array}$

$1989 \quad 22$

$1990 \quad 4 \quad 26$

$\begin{array}{lll}1991 & 4 & 27\end{array}$

$1992 \quad 1 \quad 20$

$\begin{array}{lll}\text { Total } & 18 & 149\end{array}$

\section{Graphical Techniques}

To explore the relations between subsets of the data, mark the subsets on one axis. For each of these subsets of the data, plot an estimate of $p$ and a confidence interval for $p$ against the other axis. Patterns such as trends, outliers, or large scatter are then made visible.

In Example 2, the subsets are years. The data set from each year is to be analyzed separately, and the graph is to show an estimate and a confidence interval for each year, plotted side by side. This is shown in Figure 4. The intervals were computed by a SAS program, bconfint, given in Appendix A.

For each year, a point estimate and a $90 \%$ confidence interval for $p$ have been found. The calculations were performed just as in the example with a single population. The plot can be produced easily with a graphics package that reads text files and allows asymmetrical error bars, although a hand-drawn plot would be adequate to show the results. One year appears a bit lower than the others, but the confidence intervals make it appear that the difference could have resulted from random variation. It appears acceptable to pool the data from the six years.

Sometimes a variation of this plot is useful, in which the confidence interval for the pooled data is also shown, as in Example 3. As with the earlier examples, the counts come from a draft study which 


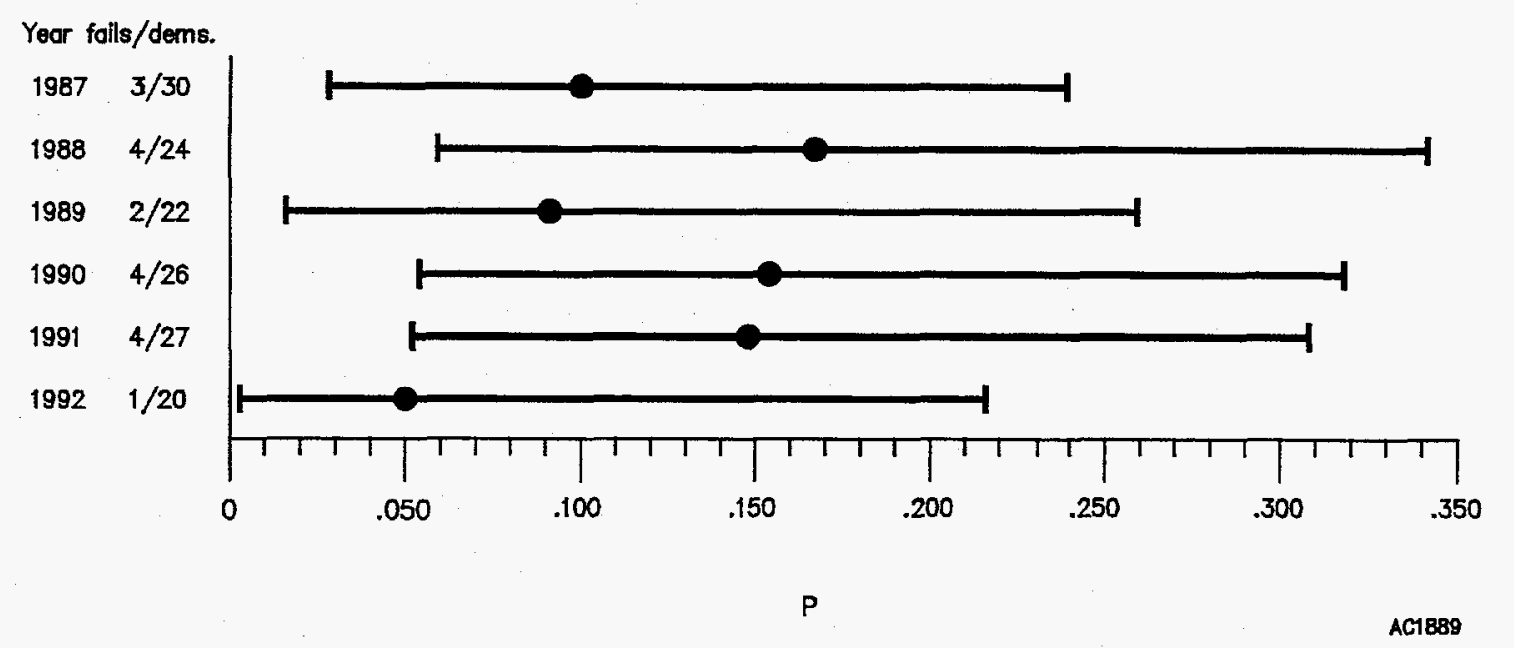

Figure 4. $90 \%$ confidence intervals for failure to start, by year.

has since been changed. Therefore, the names of the plants have been changed to generic names, to prevent anyone from drawing erroneous inferences about particular plants.

Example 3. Failures to start on demand, by plant.

The 149 demands and $18 \mathrm{HPCI}$ failures to start in Example 2 are counted by plant. The counts for the 23 plants are shown in Figure 5.

In Figure 5, the dotted reference line is drawn through the point estimate based on the pooled data. If only a few data subsets need to be compared, as in Figure 4, these embellishments are unnecessary. With many subsets, as in Figure 5, the eye tends to get lost without the reference line. The reference line has the added advantage of focusing the eye on the confidence intervals rather than the point estimates. In Figure 5, Plant $\mathrm{N}$ has the largest value of $\hat{p}$, but this is based on only one demand. The plants I and $\mathrm{L}$ are farther from the bulk of the plants, in the sense that their lower confidence bounds are farther from the reference line.

The data sources in Figure 4 are plotted in a natural order, from 1987 to 1992, while the data sources in Figure 5 are plotted in a completely arbitrary order, by alphabetical code. When the data sources have no natural order, it can be helpful to arrange them in some meaningful order. For example, the plants in Figure 5 could be arranged in order of plant age, if the analyst wants to investigate whether plant age is related to system performance. Or the plants could be ordered so that those with similar design characteristics are grouped together. One useful ordering, which does not require any information about the data sources other than the counts of hits and trials, is from worst to best. Figure 6 is such a 


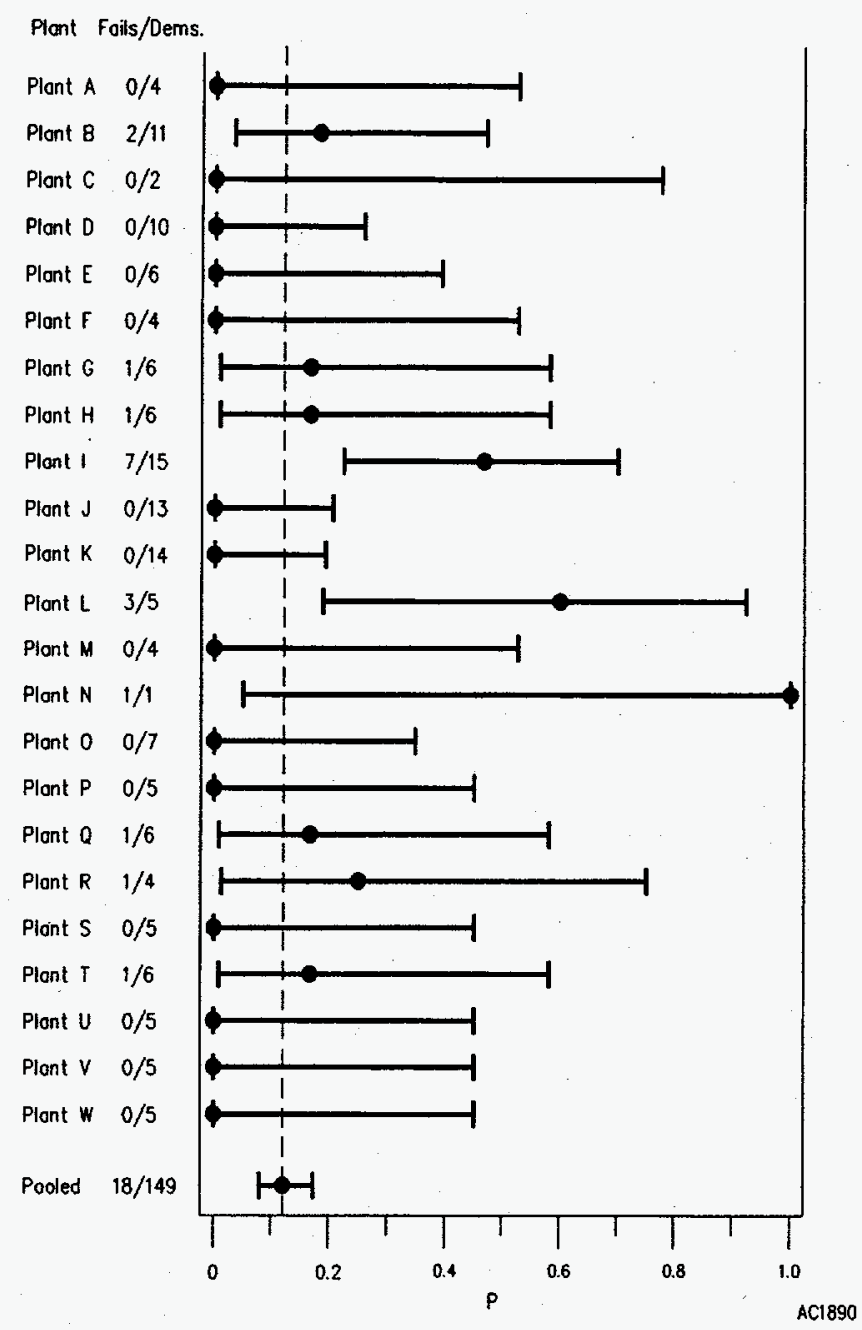

Figure 5. Failures to start 1987-92, by plant, ordered alphabetically.

graph. The rows of Figure 5 have simply been rearranged, so that $\hat{p}$ is decreasing from top to bottom. In adjacent rows with the same $\hat{p}$, the rows are arranged according to the confidence limits. This plot provides immediate answers to questions such as

Which plants performed worst in this data set?

Was the worst plant much worse than the second worst plant?

What is the median value of $\hat{p}$ ?

Cleveland (1985, Chap. 3.3) discusses this and other ways of ordering data.

These graphs are ONLY PICTURES! Pictures like these are useful, but cannot be used in an easy way to draw naive conclusions about differences between data sources. In particular: 


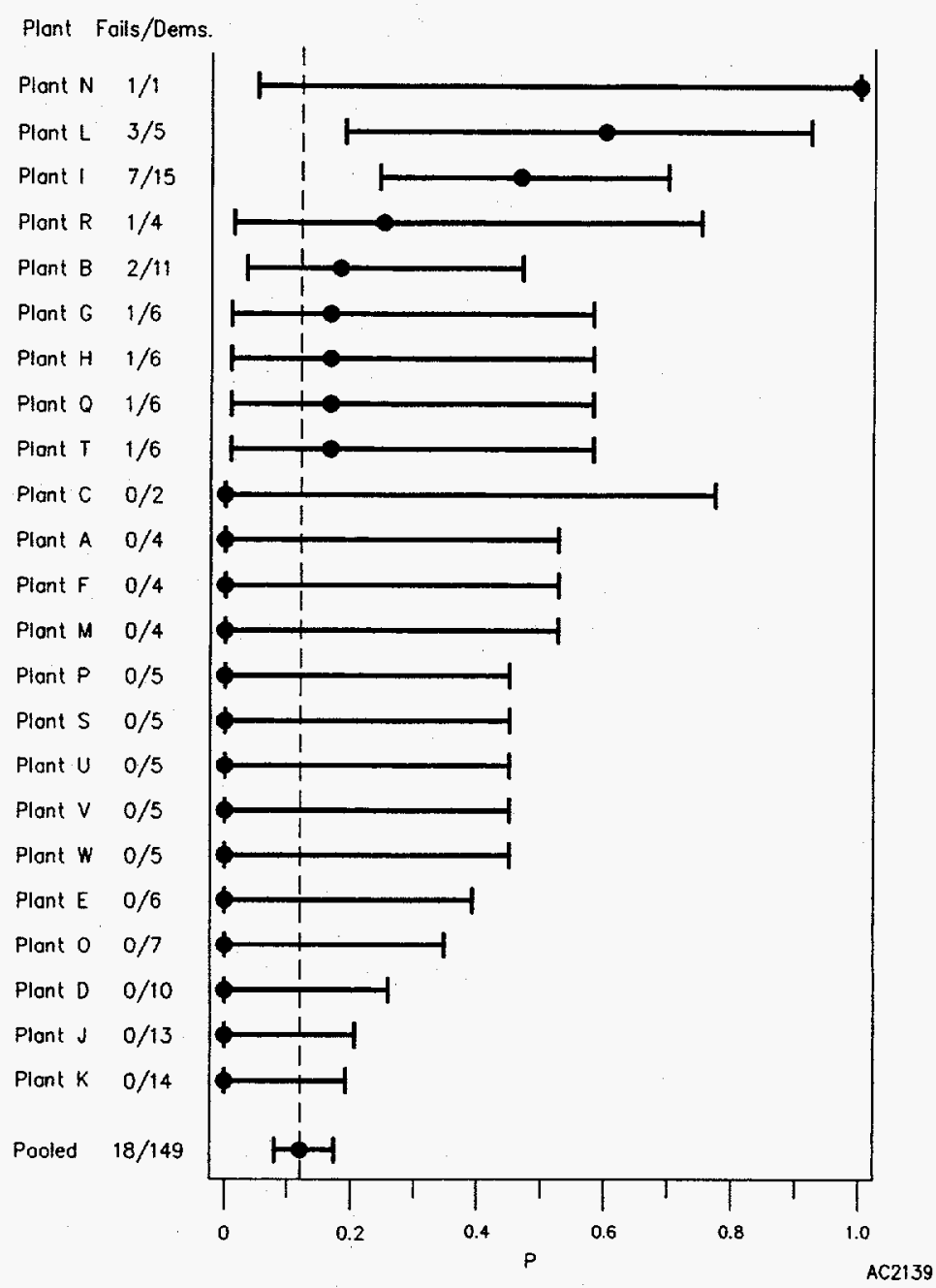

Figure 6. Failures to start $1987-92$, by plant, ordered by decreasing $\hat{p}$.

- If many confidence intervals are plotted, all based on data with the same $p$, a few will be far from the others because of randomness alone. An outlying interval does not prove that the $p$ 's are unequal.

- If there are few intervals, on the other hand, intervals that just barely overlap can give strong evidence for a difference in the $p$ 's.

To quantify the strength of the evidence seen in the picture, a formal statistical procedure is given in the next subsection. The picture gives a preview, and helps in the interpretation of the formal statistical quantification. In Example 2, if the statistical test finds a statistically significant difference between years, we naturally would then ask what kind of difference exists. The picture shows that $p$ does not steadily increase or decrease. Instead, any difference between years seems to be random bouncing of $p$, 
or possibly a one-time lower $p$. In this way, the picture provides insight even though it does not provide a quantitative statistical test.

\section{Statistical Tests}

Simple Contingency Tables $(2 \times \mathrm{J})$. The natural format for the data is a "contingency table." An introductory reference to this subject is Everitt (1992), and many general statistics texts also have a chapter on the topic. Contingency tables will be illustrated and explained here by several examples. In a two-way table, two attributes of the events are used to define rows and columns, and the numbers in the table are counts. In Example 2, two attributes of any event are its year and whether it is a failure or success. One way to build a contingency table is to let the first row show system failures and the second row system successes. Then let six columns correspond to the years 1987 through 1992 . The table entries are the counts of the events for each cell, shown in Table 1.

This is a $2 \times 6$ table, because the basic data counts occupy two rows and six columns. The row totals, column totals, and grand total are shown in the right and bottom margins. A general two-way contingency table has $I$ rows and $J$ columns. (Although this discussion is focusing on $2 \times J$ tables, with $I \times J$ tables deferred to the next section, it is simpler to give the general formulas now, keeping in mind that the example of this section has $I=2$.) The count in the $i$ th row and $j$ th column is denoted $n_{i j}$, for $i$ any number from 1 to $I$ and $j$ from 1 to $J$. Table 1 has $n_{13}=2$ and $n_{25}=23$. The total count in row $i$ is denoted $n_{i+}$ and the total count in column $j$ is denoted $n_{+j}$. The above example has $n_{2+}=131$ and $n_{+2}=24$. The grand total is denoted $n_{++}$, and equals 149 in the example.

In this example, let $H_{0}$ denote the hypothesis that $p$ is the same in all six years. We want to investigate whether $H_{0}$ is true. The method used is to see what kind of data would be expected when $p$ really is the same, and then to see how much the observed counts differ from the expected. If the difference is small, the counts are consistent with the hypothesis $H_{0}$. If, instead, the difference is large, the counts show strong evidence against $H_{0}$.

Table 1. Contingency table using failures to start on demand, by year.

\begin{tabular}{lrrrrrr|r}
\hline & 1987 & 1988 & 1989 & 1990 & 1991 & 1992 & Total \\
Failures & 3 & 4 & 2 & 4 & 4 & 1 & 18 \\
Successes & 27 & 20 & 20 & 22 & 23 & 19 & 131 \\
\hline Total & 30 & 24 & 22 & 26 & 27 & 20 & 149 \\
\hline
\end{tabular}


If $H_{0}$ is true, that is, if $p$ is really the same for all the years, the natural estimate of $p$ is $\hat{p}=$ $n_{1+} / n_{++}=18 / 149$. Then in 1987 we would have expected $30 \times(18 / 149)$ failures on average, in 1988 $24 \times(18 / 149)$ failures, and so forth. This reasoning leads to the formula for the expected count in cell $i j$ :

$e_{i j}=n_{i+} n_{+j} / n_{++}$

The difference between the observed count and the expected count for any cell is $n_{i j}-e_{i j}$. There are many cells, and therefore many ways of combining the differences to yield an overall number. One useful way is to construct

$X^{2}=\Sigma_{i} \Sigma_{j}\left(n_{i j}-e_{i j}\right)^{2} / e_{i j}$

$X^{2}$ is called the chi-square statistic, or sometimes the Pearson chi-square statistic after its inventor, Karl Pearson. When $H_{0}$ is true and the total count is large, the distribution of $X^{2}$ has a distribution that is approximately $\chi^{2}$ (pronounced chi-square) with $(I-1) \times(J-1)$ degrees of freedom. In Table 1 , the number of degrees of freedom is $(2-1) \times(6-1)=5$. If $X^{2}$ is large, compared to the $\chi^{2}$ distribution, the evidence is strong that $H_{0}$ is false; the larger $X^{2}$, the stronger the evidence.

In Table 1, suppose $X^{2}$ were 11.6. A table of the $\chi^{2}$ distribution shows that 11.1 is the 95th percentile of the $\chi^{2}$ distribution with 5 degrees of freedom, and 12.8 is the 97.5 th percentile. After comparing $X^{2}$ to these values, we would conclude that the evidence is strong against $H_{0}$, but not overwhelming. Quantitatively, we would "reject $H_{0}$ at the $5 \%$ significance level, but not at the $2.5 \%$ significance level." This is sometimes phrased as "the p-value is between 0.05 and 0.025 ." If instead $X^{2}$ were 5.1 , it would lie between the 50th and the 70th percentiles of the $\chi^{2}$ distribution, and therefore would be in the range of values that would be expected under $H_{0}$. We would say "the observed counts are consistent with the hypothesis $H_{0}$," or " $H_{0}$ cannot be rejected," or "the evidence against $H_{0}$ is weak." We do not conclude that $H_{0}$ is true, because it probably is not exactly true to the tenth decimal place, but we conclude that it cannot be rejected by the data.

The above considerations are valid if the $n$ 's are "large." If the $n$ 's are small, $X^{2}$ has a discrete distribution, and so cannot have a $\chi^{2}$ distribution. As a rather extreme example, if $n_{++}$were equal to 3 in the framework of Table 1 , there would only be a few ways that the three demands (and the number of failures, at least zero and at most three) could be arranged among the six years, and therefore $X^{2}$ could only take a few possible values. Therefore, the user must ask how large a count is necessary for the $\chi^{2}$ approximation to be adequate. An overly conservative rule is that all the expected cell counts, $e_{i j}$, be 5.0 
or larger. Despite its conservatism, this rule is still widely used, and cited in the outputs of many current statistics packages, including SAS. For a $2 \times J$ table, Everitt (1992, Sec. 3.3), citing work by Lewontin and Felsenstein (1965), states that the $\chi^{2}$ approximation is adequate if all the $e_{i j}$ 's are 1.0 or greater, and that in "the majority of cases" it is sufficient for the $e_{i j}$ 's to be 0.5 or greater.

If the expected cell counts are so small that the $\chi^{2}$ approximation appears untrustworthy, the analyst has two choices. (a) Pool some columns or rows, thereby combining cells and increasing the expected cell counts. For example, Table 1 is all right as it stands, because it is a $2 \times 6$ table with every expected cell count $>2$. Nevertheless, the expected cell counts could be made all greater than 5.0 by combining 1987 and 1988 into one column, 1989 and 1990 into a second column, and 1991 and 1992 into a third. (b) Some statistical software packages, including SAS, can compute the "exact distribution" of $X^{2}$ in some cases. Conditional on the $n_{i+}$ 's and $n_{+j}$ 's, this exact distribution is the finite set of values that $X^{2}$ can possibly take, together with their associated probabilities. If the analyst is willing to base the decision on this conditional distribution, the exact distribution can be used.

Unfortunately, SAS can compute the exact distribution only for small tables; the limiting table size depends on the hardware and the version of SAS. When SAS cannot compute the exact distribution, it may try for a very long time before giving up. The commercial package StatXact (1994) performs such calculations using modern fast algorithms, although with a large table it too is limited by the available memory in the machine. When it cannot calculate the exact p-value, it can calculate a confidence interval for the exact p-value by Monte Carlo simulation. One version of StatXact can interface with SAS, producing a single output stream.

The contingency table in Table 1 was analyzed by SAS, using the program bconfint in Appendix A, and the output is shown in Table 2.

The rows of Table 2 correspond to failures and successes, respectively, and the columns correspond to the six years 1987 through 1992 . The three numbers in the $i j$ th cell are the observed count $n_{i j}$, the expected count $e_{i j}$, and the contribution to $X^{2},\left(n_{i j}-e_{i j}\right)^{2} / e_{i j}$, respectively. The largest contribution to $X^{2}$ is 0.83 , for the failures in 1992 . This property of 1992 is consistent with the plot shown in Figure 4 . To assess the significance level, use Fisher's exact test, because the expected cell counts are too small for the chi-square distribution to be accurate. By the exact test, the tail probability beyond the observed $X^{2}=2.184$ is 0.839 , which is much too large to warrant rejection of the hypothesis of independence of rows and columns. (Using the $\chi^{2}$ approximation, the tail probability, or p-value, is 0.823 , which would lead to the same conclusion.) We conclude that there is no evidence of a difference between years, just as we believed after looking at Figure 4. 
Table 2. Analysis of failures to start on demand, by year.

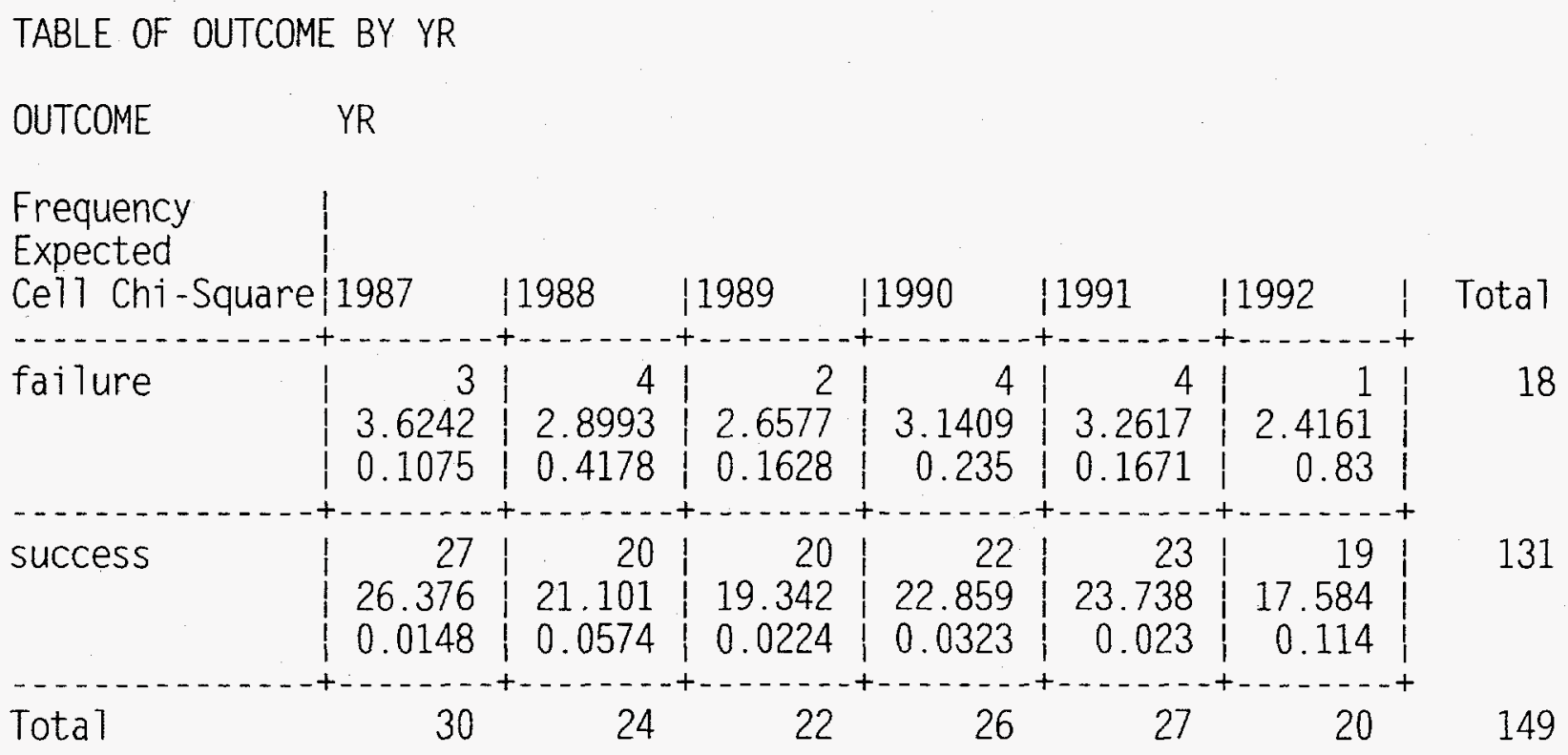

STATISTICS FOR TABLE OF OUTCOME BY YR

$\begin{array}{lccr}\text { Statistic } & \text { DF } & \text { Value } & \text { Prob } \\ \text { Chi-Square } & 5 & 2.184 & 0.823 \\ \text { Likelihood Ratio Chi-Square } & 5 & 2.368 & 0.796 \\ \text { Mante1-Haenszel Chi-Square } & 1 & 0.067 & 0.796 \\ \text { Fisher's Exact Test (2-Tai1) } & & 0.839 \\ \text { Phi Coefficient } & 0.121 & \\ \text { Contingency Coefficient } & 0.120 \\ \text { Cramer's V } & 0.121 \\ \text { Sample Size = 149 } & \\ \text { WARNING: 50\% of the cel1s have expected counts less } \\ \quad \text { than 5. Chi-Square may not be a valid test. }\end{array}$

For the data of Figure 5, the conclusions are different. The contingency table has dimensions $2 \times 23$, because the study uses 23 plants. This leads to lengthy SAS output, described here but not shown. The observed $X^{2}$ is 48.70 . The probability $P\left(\right.$ a $\chi^{2}$ random variable with 22 degrees of freedom is greater that 48.7 ) is 0.001 . Therefore, we reject the hypothesis that the probability of failure to start is the same at all plants. This calculation is based on the $\chi^{2}$ distribution for $X^{2}$, an approximation.

Some experimenting with exact calculations yielded the following results. SAS was unable to obtain the exact probability on a 386 IBM PS/2 with 18 megabytes of RAM running with DOS 6.0. On a DEC 
server 5500 with 64 megabytes of RAM running under 4.3 Ultrix, SAS calculated the exact probability to be 0.00192 . StatXact was run on a machine with 2 megabytes of RAM, which was not quite enough memory to allow it to calculate the exact probability. However, StatXact used simulation and found a $99 \%$ confidence interval for the probability to be $(0.0005,0.0017)$. Although the SAS and StatXact exact calculations conflict to some extent (possibly because of accumulated roundoff), either calculated probability is small enough to cause us to reject the hypothesis that the probability of failure to start is the same at all plants.

The largest contributors to $X^{2}$ are 14.85 from Plant $\mathrm{I}, 9.50$ from Plant $\mathrm{L}$, and 6.40 from Plant N. Therefore the three plants that are farthest from the bulk of the data are those that stand out in Figures 5 and 6 . The three $\chi^{2}$ contributions have the same ranking as the lower ends of the confidence intervals; this is often the case.

This $\chi^{2}$ method does not use any ordering of the data subsets. In Example 2, the ordering is natural, the chronological order from 1987 through 1992. However, in Example 3 the order is arbitrary, an alphabetical order of plant identifiers. The analysis method is exactly the same in the two cases, ignoring any natural order that may be present. When the data subsets have a meaningful order, it may be useful to perform additional analyses. The analysis given above is valid, but an additional possible analysis, making use of time order, is considered in the later subsection that considers trials in sequence. The additional possible analysis that can be performed with a $2 \times 2$ table, where only two possible orders exist for the two data subsets, is considered next.

Special Considerations for $2 \times 2$ Tables. The null hypothesis, $H_{0}$, states that all the data sources have the same $p$, and the alternative hypothesis, $H_{1}$, states that they do not all have the same $p$. In a $2 \times J$ table, $H_{1}$ can take many forms, because any of the $J$ data sources can have the largest or smallest $p$. In a $2 \times 2$ table, $H_{1}$ has a much simpler structure: the first data source has either the larger $p$ or the smaller $p$. These are the two cases for $H_{1}$. It is therefore possible to consider one-sided alternative hypotheses, and test $H_{0}$ against the hypothesis that the first data source has (say) the larger $p$.

The $2 \times 2$ table not only has a simple structure for the alternative hypothesis; it also has a simple structure for the data. Treat the marginal totals $n_{1+}, n_{2+}, n_{+1}$, and $n_{+2}$ as known, either because they are known from the structure of the problem or because we condition on them. Then any one of the $n_{i j}$ 's, such as $n_{11}$, determines the other three, and therefore determines the value of $X^{2}$. Therefore, $n_{11}$ may be used instead of $X^{2}$ as the test statistic. The distribution is hypergeometric, and this fact is the basis of Fisher's exact test in a $2 \times 2$ table. This allows for one-sided tests corresponding to the one-sided hypotheses mentioned in the previous paragraph. For example, suppose that row 1 corresponds to hits, 
and columns 1 and 2 correspond to data sources. Let $p_{j}$ denote the probability of a hit for data source j. To test

$H_{0}: p_{1}=p_{2}$

against the one-sided alternative hypothesis

$H_{1}: p_{1}>p_{2}$,

reject $H_{0}$ if $n_{11}$ is large. How large depends on the distribution of $n_{11}$ when $H_{0}$ is true, and what the Type I error of the test should be. For $2 \times 2$ tables, SAS always prints the p-values for the left, right, and two-sided exact tests.

A $2 \times 2$ setup is illustrated by Example 4 .

Example 4. Failures to recover from failure to start.

The $18 \mathrm{HPCI}$ failures to start (FTS's) occurred on two types of actuations. Six were on unplanned actuations, and the operator recovered the system (successfully started it manually) in all 6 cases. The remaining 12 FTS's were on periodic surveillance tests, and the operator recovered the system in only 2 of the 12 cases.

For this example, we ask if a successful recovery from FTS is as likely during an unplanned actuation as during a surveillance test. To answer this question, let $p$ be the probability of failure to recover, given FTS. Table 3 shows the SAS output. The cells termed "failure" refer to failure to recover, not FTS.

Most of the SAS printout is like that of Table 2. Note, however, that three exact tests are given: the left, right, and two-sided. From thinking about what it would make sense to reject, we see that the "left" test must correspond to the alternative hypothesis that recoveries have higher probability on unplanned actuations, and the "right" test must correspond to the alternative hypothesis that recoveries have higher probability on surveillance tests. If, without being influenced by the data, the analysts had been convinced that a one-sided alternative hypothesis was of concern, then the appropriate one-sided test should have been used. In fact, the analysts initially could imagine reasons for either ordering, so they used the two-sided test. Because they rejected the hypothesis of equal $p$ for the two types of actuations, they did not pool the data in their subsequent analyses, but instead used only the data from unplanned 
Table 3. Failures to recover from FTS, by actuation type.

TABLE OF OUTCOME BY ACTUTYPE

\begin{tabular}{|c|c|c|c|}
\hline $\begin{array}{l}\text { OUTCOME } \\
\text { Frequency } \\
\text { Expected } \\
\text { Ce11 Chi-Square }\end{array}$ & ACTUTYPE & SurvTest| & Total \\
\hline faiture & $\begin{array}{r}0 \\
3.3333 \\
3.3333\end{array}$ & $\begin{array}{r}10 \\
6.6667 \\
1.6667\end{array}$ & 10 \\
\hline CC & $\begin{array}{r}6 \\
2.6667 \\
4.1667\end{array}$ & $\begin{array}{r}2 \\
5.3333 \\
2.0833\end{array}$ & \\
\hline
\end{tabular}

STATISTICS FOR TABLE OF OUTCOME BY ACTUTY.PE Statistic DF Value Prob

Chi-Square

Likelihood Ratio Chi-Square $\quad \begin{array}{lll}1 & 13.917 & 0.000\end{array}$

Continuity Adj. Chi-Square $\quad \begin{array}{lll}1 & 8.128 & 0.004\end{array}$

Mantel-Haenszel Chi-Square $\quad \begin{array}{lll}1 & 10.625 & 0.001\end{array}$

Fisher's Exact Test (Left) 1.51E-03

(Right) $\quad 1.000$

(2-Tai1) $1.51 \mathrm{E}-03$

Phi Coefficient

$-0.791$

Contingency Coefficient $\quad 0.620$

Cramer's V

$-0.791$

Sample Size $=18$

WARNING: $50 \%$ of the cells have expected counts less

than 5 . Chi-Square may not be a valid test.

actuations to estimate the probability of recovery from FTS.

Tables with Other Dimensions. Consider now tables other than $2 \times J$. When the counts are partitioned many ways, the cell counts are often too small to yield any interesting conclusions. Nevertheless, such tables are sometimes useful. Here are some possibilities. 
In a general two-way table, an $I \times J$ table with more than two rows, the $i$ th row has probability $p_{i}$. This generalizes the $2 \times J$ table, where the first row has probability $p$ and the second probability 1-p. The hypothesis $H_{0}$ is that each $p_{i}$ is the same in all the columns. This is commonly expressed by saying that the rows and columns are independent. If $H_{0}$ is true, the expected cell count $e_{i j}$ is given by Equation (5).

A $3 \times J$ table could be formed by considering the three pressurized water reactor (PWR) vendors Babcock \& Wilcox, Combustion Engineering, and Westinghouse. The counts are all events of some type, such as all reported failures. Note, this example is not based on the failure/success dichotomy. Now consider $J$ causes of the failures, such as equipment failure, personnel error, procedural error, etc. A $3 \times J$ contingency table could be used to investigate whether vendor and failure cause are independent. $H_{0}$ would be the hypothesis that the fraction of events from personnel error (or any other cause) is the same for all three vendors.

A general $I \times J$ table could result, for example, from partitioning an observed number of failures according to a list of $I$ failure causes and $J$ years. Then $n_{i j}$ would be the number of failures from cause $i$ in year $j$.

All the above tables have two dimensions, although the number of cells varies for the different examples. Higher dimensional tables, such as $2 \times 3 \times 5$, are more complicated and not considered here. The complexity follows from the many possible hypotheses that could be tested, for example: (a) rows, columns, and layers are all independent; (b) rows depend on columns but not on layers; and (c) rows depend on columns and on layers, but not on any interaction between columns and layers. This leads to loglinear modeling, too large a topic for this report. The theory of loglinear modeling and multi-way tables is currently being vigorously developed at universities.

Finally, one-way tables $(1 \times J)$ exist, but they arise most naturally when considering events per time rather than hits per trial. Therefore, they are not discussed here.

\section{Assumption of Constant $p$ for Trials in Sequence}

Other tools are useful if the hits and misses can be arranged in a single sequence. This is possible, for example, if the date of each trial is known. 


\section{Graphical Technique}

A very basic plot is a cumulative plot of the number of hits against the number of trials. The wide variety of other possible plots is not considered in this report on basic methods.

Example 5 is a case in which the hits and misses occur in sequence. It is taken from the same data set as the other examples of this report. Because that data set has since been modified, no inferences should be drawn from the numerical conclusions here.

Example 5. Licensee Event Reports with safety function lost or available.

There were 195 Licensee Event Reports submitted for HPCI system inoperabilities in 1987-1992. Of these, 123 involved loss of the safety function. The others were violations of various technical specifications or design requirements, but did not reflect an inability of the system to function when required. The events were ordered by their dates, and the cumulative number of events with safety function lost was plotted against the event sequence number in Figure 7. 


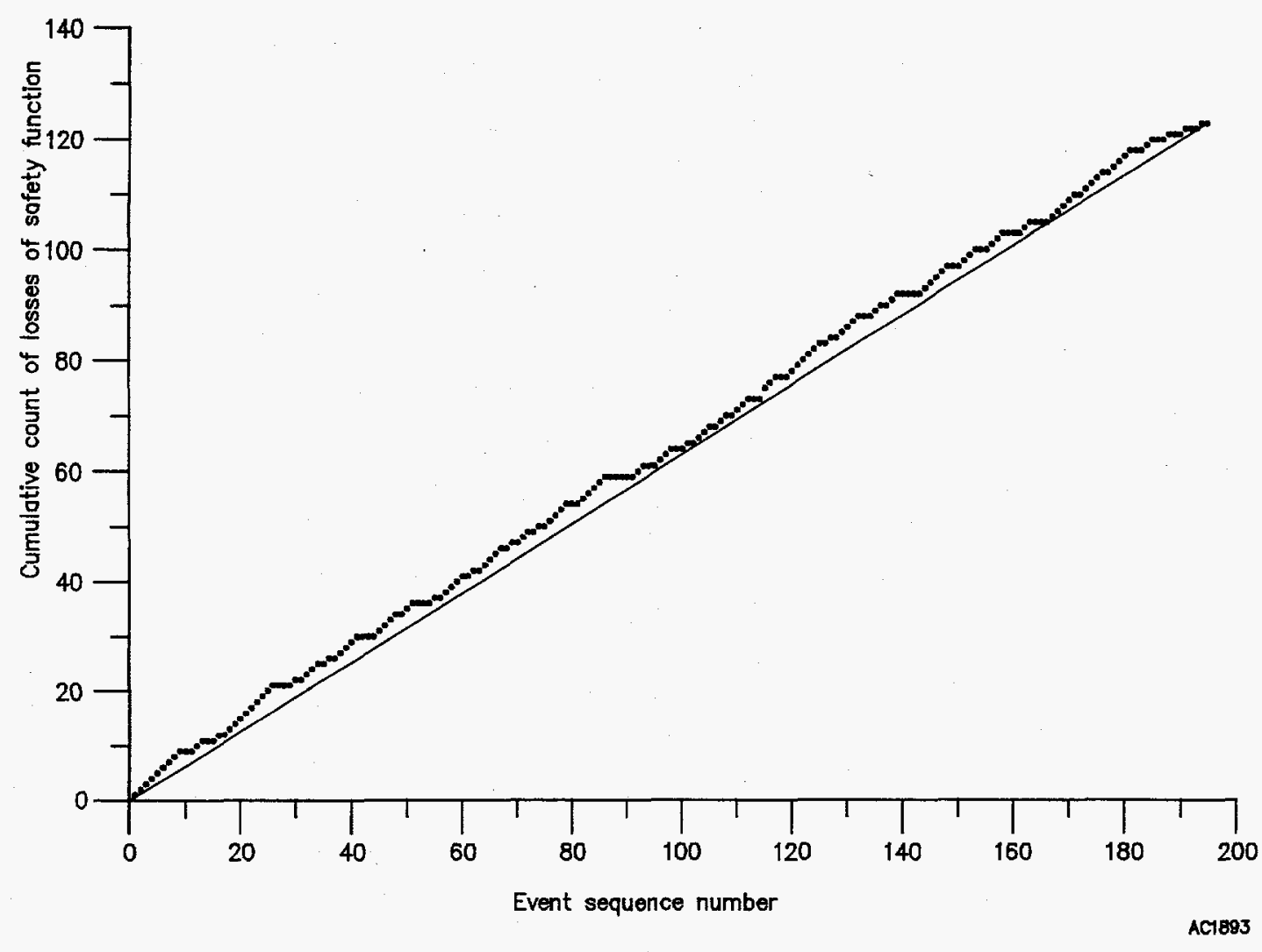

Figure 7. Cumulative count of losses of safety function, among all reported events.

In this example, the Licensee Event Reports are the "trials," and the events with safety function lost are the "hits." In Figure 7, the dots show the cumulative count of hits. For example, the first nine reports (sequenced according to date) described events when the safety function was lost. Therefore, the first nine dots in the plot are at $(1,1),(2,2), \ldots,(9,9)$. The next two reports described events when the safety function was not lost, so the next two dots in the plot are at $(10,9)$, and $(11,9)$. The dots rise to the final value of $(195,123)$.

A solid diagonal reference line is also shown in Figure 7 . The left end point is at $(0,0)$ and the right end is at $(195,123)$. Because the dots stay close to the line, we conclude that the frequency of losses of safety function within the reports does not appear to change. Of course, a formal statistical test is needed to confirm this visual impression.

The details of the diagonal line deserve a little discussion. Why not use a least squares fit, or some other fitted line? The line is intended to estimate the expected height of the plot at any horizontal point. After $n$ trials, the expected number of hits is $\hat{p} n=(123 / 195) n$. This line starts at $(0,0)$, and rises to height 123 when $n=195$. Therefore, this line is plotted, not some other. 


\section{Statistical Test}

Continuing with this example, consider the null hypothesis

$H_{0}: p$, the probability of a hit, is the same for all trials.

This says that the hits are uniformly distributed within the sequence of trials.

There are several tests of $H_{0}$. The one to choose depends on the possible alternative distributions that should be detected. Against the alternate hypothesis that the hits tend to occur more at one end of the sequence (that is, $p$ is either an increasing or a decreasing function of the sequence number), use the Wilcoxon-Mann-Whitney test, described in texts that cover nonparametric statistics. One good source of standard nonparametric methods is Hollander and Wolfe (1973).

This test is based on the sum of the ranks of the hits. For example, in the sequence of hits and misses

hit, miss, hit, hit, miss

the three hits have ranks 1,3 , and 4 , and the sum of their ranks is 8 . Let $W$ denote the sum of the ranks of $k$ hits in $n$ trials. If $k$ and $n-k$ are both large and if the probability of a hit is the same for the entire sequence, $W$ is approximately normal with mean $\mu_{\mathrm{W}}=k(n+1) / 2$ and variance $\sigma^{2}{ }_{\mathrm{W}}=k(n-k)(n+1) / 12$. If $Z=\left(W+0.5-\mu_{\mathrm{W}}\right) / \sigma_{\mathrm{W}}$ is in either tail of the distribution, the null hypothesis should be rejected; the value 0.5 is a continuity correction. If $k$ or $n-k$ is small, statistics books give tables, or statistical computer packages calculate the exact tail probability.

The data of Figure 7 were written as a text file with each line containing a date and an indicator variable for whether the safety function was lost or not. This was read by SAS. Each date was converted to the Julian date, which have the form YYDDD, two digits for the year followed by three digits for the day ( 1 to 366 ). This form was chosen so that later dates automatically correspond to larger numbers. Conversion to the format YYMMDD would also have accomplished the goal of ordering the events in chronological sequence.

The data of Figure 7 were analyzed this way by the SAS program WMW, given in Appendix A. The output is shown in Table 4. The labels SFL and SFA mean "safety function lost" and "safety function available." 
Table 4. Wilcoxon-Mann-Whitney test of constant hit probability in Licensee Event Reports.

NPAR 1 WAY PROCEDURE

Wilcoxon Scores (Rank Sums) for Variable DATE

Classified by Variable CATEGORY

\begin{tabular}{|c|c|c|c|c|c|c|}
\hline CATEGORY & $N$ & $\begin{array}{l}\text { Sum of } \\
\text { Scores }\end{array}$ & $\begin{array}{l}\text { Expected } \\
\text { Under HO }\end{array}$ & $\begin{array}{l}\text { Std Dev } \\
\text { Under HO }\end{array}$ & $\begin{array}{l}\text { Mear } \\
\text { Score }\end{array}$ & \\
\hline $\begin{array}{l}\text { SFL } \\
\text { SFA }\end{array}$ & $\begin{array}{r}123 \\
72\end{array}$ & $\begin{array}{r}11539.0 \\
7571.0\end{array}$ & $\begin{array}{r}12054.0 \\
7056.0\end{array}$ & $\begin{array}{l}380.325406 \\
380.325406\end{array}$ & $\begin{array}{r}93.813008 \\
105.152778\end{array}$ & \\
\hline $\begin{array}{l}\text { Average } \\
\text { Wi } 1 \text { coxon } \\
\text { (with Cor }\end{array}$ & $\begin{array}{l}\text { res } \\
\text { Samp } \\
\text { nuity }\end{array}$ & $\begin{array}{l}\text { used for } \\
\text { st (Norma } \\
\text { rection o }\end{array}$ & proximatic & & & \\
\hline$S=7571$. & & & 1.35279 & & $o b>|z|=$ & 0.1761 \\
\hline T-Test ap & $0 x .5$ & ficance $=$ & 0.1777 & & & \\
\hline $\begin{array}{l}\text { KruskaT-h } \\
\text { CHISQ }=1\end{array}$ & $\begin{array}{l}\text { is } \\
36\end{array}$ & (Chi -Squ & $\begin{array}{l}\text { pproximat } \\
=1\end{array}$ & Prob & $>\mathrm{CHISQ}=$ & 0.1757 \\
\hline
\end{tabular}

In Table 4, the value of $Z$ is 1.35 , which is not in the extreme tail of a normal distribution. Therefore, as we expected from viewing Figure 7, the test shows no strong evidence of an increasing or a decreasing trend in $p$. In Figure 7 , the observed cumulative hit count is above and to the left of the straight line. Interpretation of the difference as "to the left" suggests that the sum of the hit (SFL) ranks will be less than the expected value, just as observed in Table 4.

Note, although the sum of the SFL ranks is less than the expected value, the calculated $Z$ is positive. This is because SAS calculates $Z$ for the category with the smaller sum of ranks, SFA in this example. The absolute value of $\mathrm{Z}$ is the same (except for the small continuity correction) whether it is based on SFL or SFA ranks, so a two-sided test reaches the same conclusion either way. To interpret the results correctly for a one-sided test, however, look at the printed rank sums, not at the sign of $Z$. 


\section{Assumption of Independent Trials}

The preceding subsections presented ways to investigate whether $p$ is the same for all trials. Now consider the other major assumption of the simple binomial model, that the outcomes of different trials are statistically independent.

Trials can be dependent in many ways, and some of them must be addressed by careful thinking rather than by statistical data analysis. For example, if different pieces of hardware are demanded separately, their failures can be presumed to be independent unless a common cause synchronizes the failures of pieces of hardware. (See Atwood 1986, Mosleh et al. 1987, or Siu and Mosleh 1989 for examples.) If common cause failures form a noticeable fraction of all the failures, the study team should probably divide the independent failures and common cause failures into separate data sets, and separately estimate the probabilities of each kind of failure.

If hits occur in sequence, it is natural to consider serial dependence, in which the occurrence of a hit on one trial influences the probability of a hit on the next trial. Some people believe that hits in baseball occur this way, that a slump or streak can persist because of a batter's attitude, which is influenced by how successful he has been recently. In the context of hardware failures, suppose failures are sometimes diagnosed incorrectly, and therefore repaired incorrectly. Immediately after any failure, the probability of failure on the next demand is higher, because the first failure cause may not have been truly corrected. In such a case, the failures would tend to cluster rather than being uniformly scattered among the successes. A cumulative plot such as that in Figure 5 can be inspected for such clusters.

A statistical test that could be applied is the Wald-Wolfowitz runs test, presented, for example, by Bain and Engelhardt (1992, Sec. 14.9) or Gibbons (1976, Sec. 8.2). A run is an uninterrupted sequence of hits or an uninterrupted sequence of misses. For example, the sequence

hit, miss, miss, hit, miss, miss, miss, hit, hit

has five runs: a run of one hit, a run of two misses, a run of one hit, a run of three misses, and a run of two hits. If a hit makes the next trial more likely to result in a hit, the hits tend to cluster, and there will be fewer runs than if the outcomes were independent. If a hit makes the next trial more likely to result in a miss, the hits and misses tend to alternate, and there will be more runs than if the outcomes were independent. Depending on which kind of serial dependence is of concern, a one-sided test or a two-sided test may be used. 
As before, let $n$ be the number of trials and $k$ the number of hits. Let $T$ be the total number of runs. It can be shown that when the trials are independent, $T$ has mean and variance

$E(T)=2 k(n-k) / n+1$

$\operatorname{var}(T)=2 k(n-k)[2 k(n-k)-n] /\left[n^{2}(n-1)\right]$

and, if $k$ and $n-k$ are greater than 10 or so, $T$ is approximately normal. Therefore, a test statistic is

$Z=[T+0.5-E(T)] /[\operatorname{var}(T)]^{1 / 2}$

The 0.5 is a correction for continuity, especially useful when the sample is too small for $T$ to be considered normal, but valid for any sample. Then $Z$ is approximately standard normal, unless the sample is very small. For very small samples, use Table XX of Walpole and Myers (1985), Table L of Gibbons (1976), or the exact formulas given by Bain and Engelhardt.

The runs test could also be used to test for a trend, because putting the hits at one end of the sequence produces fewer runs than if the hits were randomly scattered. However, the Wilcoxon-MannWhitney test is more powerful than the runs test for detecting a trend, and therefore is preferred when testing for a trend. Gibbons (1976) states "Runs test for randomness are not particularly powerful, mainly because of the generality of the inference situation and the very weak assumptions required for validity. However, they occupy a unique position in statistical inference as the only tests that focus on the order in a sequence." Therefore, the runs test is recommended only when testing for serial dependence.

We have no reason to expect the losses of safety function in Figure 7 to cluster, because the events come from many plants around the U.S. However, we use it as an example to illustrate the method. Inspection of Figure 7 reveals 84 runs, shown as 42 sloping portions of the plot and 42 level portions. The above formulas reduce to

$$
\begin{aligned}
& E(T)=2 \times 123 \times 72 / 195+1=91.831 \\
& \operatorname{var}(T)=2 \times 123 \times 72[2 \times 123 \times 72-195] /\left[195^{2} \times 194\right]=42.059=6.485^{2} \\
& Z=(84.5-91.831) / 6.485=-1.13
\end{aligned}
$$

Because $Z$ is not in the extreme tail of the standard normal distribution, we conclude that there is no evidence of the kind of serial dependence that could affect the number of runs. 


\section{MODELING VARIATION BETWEEN DATA SOURCES}

Suppose that $p$ is believed to vary. For example, the chi-square test may have rejected the hypothesis of a constant $p$, or engineering considerations may suggest that $p$ is not constant. What model should be used? The case when the data sources are sequenced and $p$ is an increasing or decreasing function of the sequence number should be treated with loglinear models, and therefore is postponed until a later report. Consider here the case for which there are a number of distinct data sources, in no particular order, and suppose that the chi-square test has rejected the hypothesis that $p$ is the same for all the data sources.

One approach is to analyze each data source separately, resulting in a separate estimate of $p$ for each data source. This is reasonable if there are only two data sources. It might also be reasonable if one data source (such as one plant) is clearly different from all the others. In this case, the one plant could be analyzed separately, and all the other sources might be considered as a single homogeneous source. After this regrouping of the data, only two sources exist, to be analyzed separately.

Often, however, removal of one outlier leaves a data set that contains another outlier, and removal of that may leave a set with still another outlier. In such cases it is simpler (that is, it involves fewer parameters to estimate) to use the following compound model. For concreteness, the model is explained here for Example 3, where the distinct data sources are plants.

\section{Beta-Binomial Model}

The method models the variation between the plants. It is assumed that $p$ varies from plant to plant, and follows a beta $(\alpha, \beta)$ distribution. Let $p_{i}$ denote the value corresponding to the $i$ th plant. At this plant, conditional on the value of $p_{i}$, it is assumed that each trial results in a hit with probability $p_{i}$. In short, the number of hits in $n$ trials at the $i$ th plant is $\operatorname{binomial}\left(n, p_{i}\right)$; the number of hits in $n$ trials at a single random plant has a beta-binomial $(n, \alpha, \beta)$ distribution. The value of $n$ is known, and the two parameters $\alpha$ and $\beta$ are unknown.

Several methods have been proposed to estimate $\alpha$ and $\beta$. Martz and Waller (1991, Section 7.6) give formulas for moment estimators. Alternatively, the maximum likelihood estimates (MLEs) could be found. They are recommended here because maximum likelihood estimation is known to be asymptotically optimal (for large samples) and because their theoretical properties are well known. In particular, the asymptotic covariance matrix is known, allowing easy implementation of the Kass-Steffey variance adjustment described below and in Appendix B. The likelihood equations are given by Martz 
and Waller, and in Appendix B. No closed-form solution exists for the MLEs of $\alpha$ and $\beta$. A numerical iteration method in the SAS language is given in Appendix A as the macro bbinmle. It is explained in Appendix B. Similar programs could be written in other languages.

The iterative procedure does not always converge. In some cases, the search for the maximum likelihood leads to $\alpha /(\alpha+\beta)$ stabilizing at a finite value, but $\alpha+\beta$ diverging to infinity. In such a case, the parametric empirical Bayes method outlined here cannot be used. One practical guide is to stop a numerical search whenever $\alpha+\beta$ appears to be larger than the total number of trials in the data set; such a large value for $\alpha+\beta$ should not be used because it would result in plant-specific intervals shorter than the interval based on simply pooling all the data.

Let us apply this to the data presented in Figure 5. If the data from all the plants were pooled, the Bayes posterior distribution based on the noninformative prior would be beta $(18.5,131.5)$, with mean 0.123 and variance 0.00072 , from Equations (1a) and (1b). A Bayes $90 \%$ interval based on this distribution is $(0.082,0.170)$. However the chi-square test says that the plants do not all have a common p. Therefore, we try the compound beta-binomial model.

The maximum likelihood estimates are $a=0.506$ and $b=3.84$, as found using the SAS macro bbinmle. The beta $(a, b)$ distribution estimates the variation of $p$ among the plants. This distribution has mean 0.116 and variance 0.0192 , from Equations (1a) and (1b). Note that the mean is similar to the estimate based on pooling the data, but the variance is much larger. A $90 \%$ interval based on this distribution is $(0.001,0.415)$, much wider than the interval based on pooling the data.

\section{Empirical Bayes Estimates}

Having estimated the overall distribution of $p$, we now naturally inquire about the value of $p_{i}$ at the $i$ th plant. The model says that $p_{i}$ was randomly selected from the beta $(\alpha, \beta)$ distribution, and the $k_{i}$ failures in $n_{i}$ demands were generated from a binomial $\left(n_{i}, p_{i}\right)$ distribution. Recall that $\alpha$ and $\beta$ are estimated by $a$ and $b$. The simplest approach is to act as if the estimates were the true values, and let the prior distribution of $p_{i}$ be beta $(a, b)$. For the $i$ th data source, let the prior distribution of $p_{i}$ be beta( $(a$, $b$ ), and find the posterior distribution of $p_{i}$ by updating the prior with the data. This is the usual Bayes method for estimating $p_{i}$, except that the prior parameters are estimated from all the data. This leads to the name "empirical Bayes estimation."

More sophisticated methods account for the variability in the estimators $a$ and $b$. In particular, Kass

and Steffey (1989) give a simple first-order correction, which is applied to the beta-binomial model by 
Martz, Abramson, and Kvam (1994). This approach leaves each plant-specific mean unchanged but lengthens the interval somewhat. A SAS program to implement the method is given in Appendix A as bbempb. The details of the method are explained in Appendix B. For most plants in the example data, this adjustment lengthens the $90 \%$ interval by only a few percent, but for Plant $L$ the adjustment lengthens the interval by $17 \%$.

Before showing a graph of empirical Bayes results, we first give a graph of ordinary (non-empirical) Bayes results. Figure 8 shows the Bayes mean and 90\% interval for each plant, based on the noninformative prior distribution and the plant-specific data. This figure is the direct analogue of Figure 6, with Bayesian estimates replacing the MLE and confidence interval. In both figures, each plant's estimates are based only on data for that plant. Figures 6 and 8 appear similar, although the Bayes point estimates are pulled from the MLEs toward the prior mean of 0.5 , and the Bayes intervals are somewhat shorter than the corresponding confidence intervals.

Figure 8 is given as an illustration, not as a method intended to compete with the empirical Bayes method. Only if the data sources are believed to have no relation to each other (such as data from different kinds of components, for example), would separate analyses like those of Figure 8 be used. If the data sources (plants, in this example) are all believed to behave as if their p's came randomly from some population, and if no prior reason exists to assign any particular $p$ to any particular data source, then the empirical Bayes method should be used.

Figure 9 shows the empirical Bayes estimates. The intervals do not vary as much from each other as in Figure 8. Instead, each plant is pulled somewhat strongly toward the overall average given by the empirical Bayes prior mean.

For example, Plant I had 7 failures in 15 trials. Therefore (ignoring the adjustment for uncertainty in $a$ and $b$ ) its empirical Bayes distribution is beta with parameters $a+7=7.506$ and $b+8=11.84$. Therefore, the point estimate of $p_{\text {Plant I }}$ is $7.506 /(7.506+11.84)=0.388$, as shown in Figure 9. The empirical Bayes mean is pulled from the simple estimate, $7 / 15=0.467$, toward the prior (or overall) mean of 0.116. The empirical Bayes method sees two causes for the high failure counts at some plants: high values of $p$, and bad luck on top of that. The empirical Bayes estimate of 0.388 corresponds to $p$ alone, and the difference $(0.467-0.388)$ is attributed to bad luck. Similarly, Plant $\mathrm{K}$ has an empirical Bayes mean of 0.028 , although the simple estimate is $0 / 14=0$. The value 0.028 corresponds to the plant alone, and the difference between that and zero is attributed to good luck. 


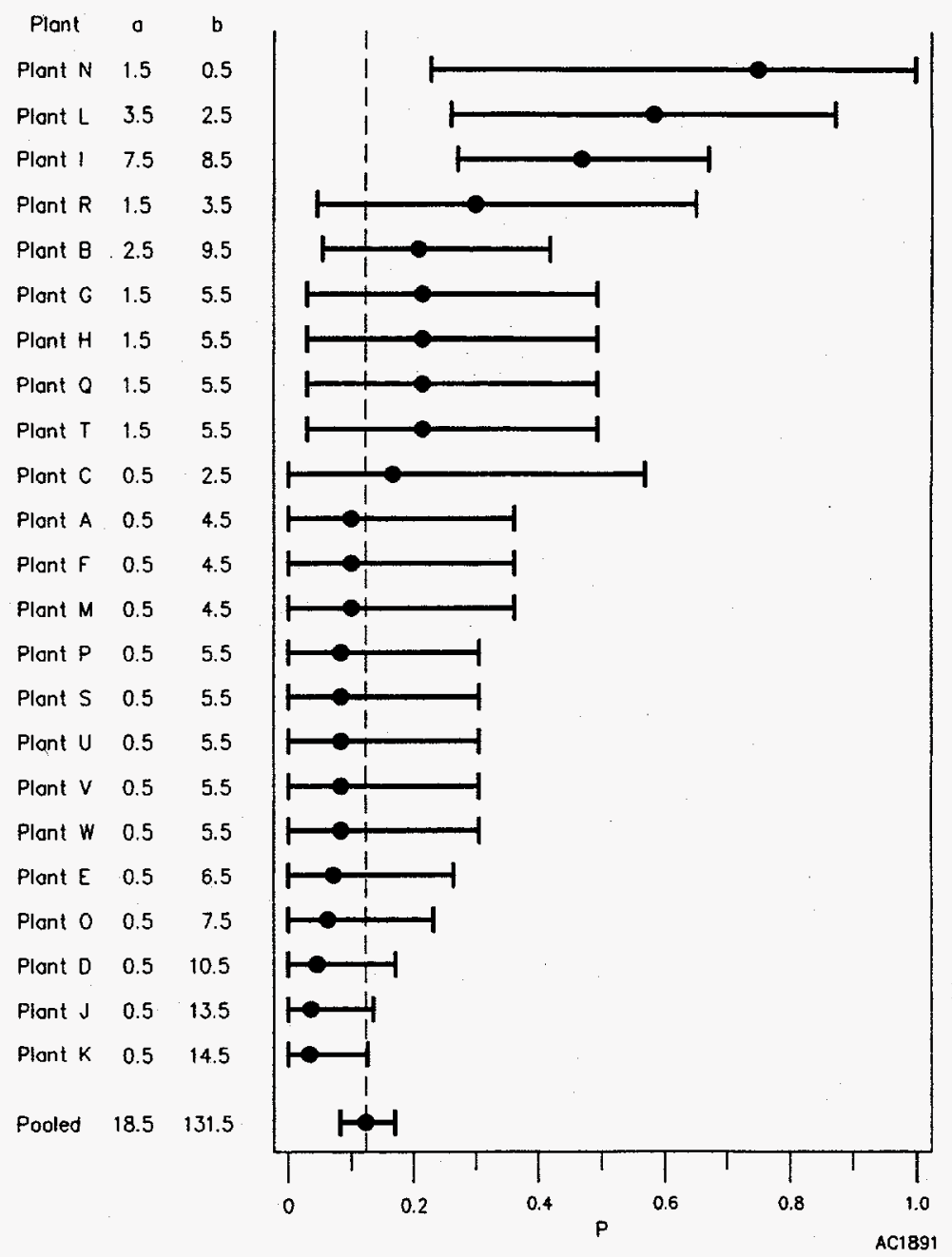

Figure 8. Bayes means and $90 \%$ intervals for $p_{i}$, with each plant estimate based on a noninformative prior distribution. Plants are ordered as in Figure 6.

Two points deserve mention. First, the empirical Bayes method only models, or quantifies, the variation. It does not explain the causes. The poor showing of Plant I may result from worse procedures, worse equipment, or some other cause. Such an investigation goes beyond the scope of the empirical Bayes data analysis.

Second, we should ask whether the variation was modeled correctly. The method assumed a beta prior distribution and then mechanically found plant-specific posterior means and intervals. Does the assumed model fit the data adequately?

One way to address this question is to ask whether the data counts are consistent with the assumed beta-binomial model. Consider Plant $I$ as an example. The beta-binomial probability of 7 failures in 15 demands is given as $P(7)$ by Equation (4) in Appendix B. The probability of 7 or more failures is $\Sigma_{k} P(k)$ 


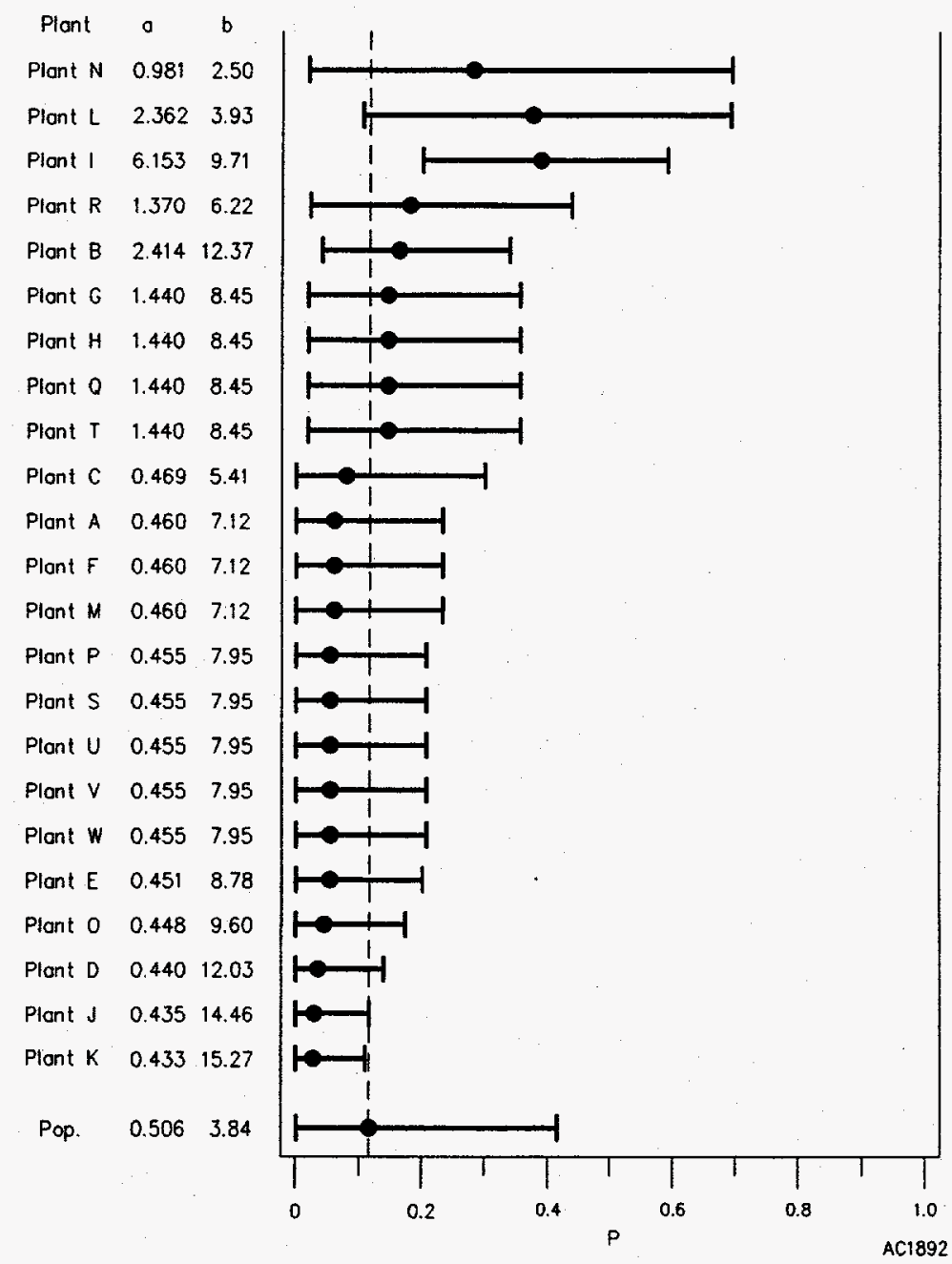

Figure 9. Empirical Bayes means and $90 \%$ intervals for $p_{i}$ by plant. Plants are ordered as in Figure 6.

where $k$ runs from 7 to 15 . The SAS macro bbempb used the estimates $a$ and $b$ for the unknown $\alpha$ and $\beta$, and evaluated the probability of 7 or more failures as 0.057 . This is a p-value, the probability that the data would appear as extreme as observed if the model assumptions are correct. From 20 plants, we would expect about one plant to have a p-value as small as $1 / 20=0.050$. In fact, from 23 plants, two (Plants I and L) have p-values as small as 0.057 . The technical details are more complex than indicated here, but it appears that there is no strong evidence against the assumption of a beta prior. One small modification, not implemented in bbempb, would be to compute each plant's p-value based on the prior distribution estimated using all plants except the one whose p-value is being computed. Finally, Martz, Abramson, and Kvam (1994) have a quite different approach to dealing with this issue. 


\section{REVIEW AND GUIDELINES}

This report consists primarily of a collection of tools that can be applied as needed, not a set of procedures that tell the reader what to do. Now that the tools have been presented, however, it is appropriate to review them and to give some general guidelines on when to use them. This also helps to show how they relate to each other.

\section{The Simple Model and Its Data Analysis}

The simple binomial model makes some basic assumptions: each trial is a hit with probability $p$-the same probability for all the trials-and a miss otherwise; the outcomes on different trials are statistically independent; the number of trials and the number of hits that occurred are both known. The analyst must think about the meaning of these assumptions for the particular data set under consideration. Much of the effort in data analysis involves checking the assumptions and adapting the methods to match the assumptions that seem to be true.

If the assumptions of the simple binomial model are believed to be true, the main issue is to estimate p. Formulas have been given for the point estimate (the maximum likelihood estimate) and confidence interval. A SAS macro bconfint has also been given to calculate these. If preferred, a Bayesian distribution may be found, yielding a point estimate (the mean of the distribution) and a Bayes interval. The SAS macro bbayes calculates these. As long as the Bayesian analysis uses the noninformative prior, the confidence interval and Bayes interval will be similar, though not identical.

Which analysis to use is partly a matter of the analyst's and customer's philosophy and taste. When $n$ is not very large, the Bayes intervals will be visibly pulled towards the prior mean, 0.5 for a noninformative prior; this is especially noticeable when the number of hits, $k$, is near 0 or $n$. When $k$ equals 0 or $n$, the confidence interval has one end at 0.0 or 1.0 , while the Bayes interval is always strictly between 0.0 and 1.0. Some people may prefer one method or the other because of these considerations.

The choice of Bayesian or non-Bayesian analysis is also partly a matter of simplicity. Confidence intervals are related naturally to hypothesis tests, and are therefore natural (though not necessary) to use when checking the model assumptions. Most PRA work requires a Bayesian approach, and therefore the final product of a PRA-oriented analysis may be a Bayesian distribution. To propagate the uncertainty in $p$ through a complicated system, PRA computer codes such as IRRAS (Russell et al. 1992, 1993) use Monte Carlo simulation, sampling $p$ from its Bayes distribution. The required inputs for IRRAS, based on the posterior distribution, have been given in this report whenever the posterior distribution is found. 


\section{Checking the Assumptions of the Simple Model}

To check the assumptions of the simple model, graphical methods and statistical tests have been presented. Three questions were addressed:

- Is $p$ the same for a number of distinct data sources?

- Is $p$ constant during an ordered (typically chronological) sequence of trials?

- $\quad$ Are the trials statistically independent?

For the first question, the methods given were a plot of side-by-side confidence intervals, and chisquare tests with $2 \times J$ contingency tables. The SAS macro bconfint performs all the necessary calculations. Contingency tables with higher dimensions were mentioned, but deferred to a later report. For the second question, the methods given were a cumulative plot of hits, and the Wilcoxon-MannWhitney test. The SAS program WMW illustrates how to conduct the test. The third question may only require careful thinking, not statistical analysis. However, the runs test was presented as a test for serial dependence.

\section{An Extension of the Simple Binomial Model}

Suppose there are distinct, but inherently similar, data sources, such as systems of similar design at different facilities. If a chi-square test shows that the data sources have different values of $p$, this variation can often be modeled by assuming that $p$ has a beta distribution. The parameters of this distribution can be estimated by maximum likelihood; the SAS macro bbinmle can be used for this. Once the beta distribution on $p$ has been estimated, it can be used as a prior distribution, and updated by the data from each data source to yield a different posterior distribution for each of the different data sources. This is a simple implementation of the empirical Bayes method. A more refined implementation, carried out by the SAS macro bbempb, accounts for the uncertainty in the parameter estimates, yielding slightly longer intervals. It also investigates whether any plants are not adequately fitted by the beta-binomial model.

This report does not consider more complicated models, such as modeling a trend in $p$ over time. 


\section{REFERENCES}

Atwood, Corwin L., 1986, “The Binomial Failure Rate Common Cause Model,” Technometrics, 28, pp. 139-148.

Atwood, C. L., C. D. Gentillon, G. M. Grant, and M. B. Sattison, 1993, High Pressure Coolant Injection (HPCI) System Performance, 1987-1992, DRAFT, EGG-ERTP-10929.

Bain, Lee J., and Max Engelhardt, 1992, Introduction to Probability and Mathematical Statistics, Second Edition, Boston: PWS-KENT Publishing Co.

Box, George E. P., and George C. Tiao, 1973, Bayesian Inference in Statistical Analysis, Reading, MA: Addison-Wesley.

Cleveland, William S., 1985, The Elements of Graphing Data, Monterey, CA: Wadsworth Advanced Books and Software.

Everitt, B. S., 1992, The Analysis of Contingency Tables, Second Edition, London: Chapman and Hall.

Gibbons, Jean Dickinson, 1976, Nonparametric Methods for Quantitative Analysis, New York: Holt, Rinehart and Winston.

Hollander, Myles, and Douglas A. Wolfe, 1973, Nonparametric Statistical Methods, New York: John Wiley \& Sons.

Johnson, Norman L., and Samuel Kotz, 1989, Discrete Distributions, New York: John Wiley \& Sons.

Kass, Robert E. and Duane Steffey, 1989, “Approximate Bayesian Inference in Conditionally Independent Hierarchical Models (Parametric Empirical Bayes Models)," Journal of the American Statistical Association, 84, pp. 717-726.

Lewontin, R. C., and J. Felsenstein, 1965, "The Robustness of Homogeneity Tests in $2 \times N$ Tables," Biometrics, 21, pp. 19-33. 
Martz, Harry F., Lee R. Abramson, and Paul H. Kvam, 1984, "Empirical Bayes Estimation of the Reliability of Nuclear Power Plant Emergency Diesel Generators: An Expository Application," LA-UR-93-1239 Rev. 2 (submitted to Technometrics).

Martz, Harry F., and Ray A. Waller, Bayesian Reliability Analysis, Malabar, FL: Krieger, 1991, Section 7.6.

Mosleh, A., et al., 1987, Procedures for Treating Common Cause Failures in Safety and Reliability Studies, NUREG/CR-4780, U.S. Nuclear Regulatory Commission.

Russell, K. D., et al., 1992, Integrated Reliability and Risk Analysis System (IRRAS) Version 4.0: Reference Manual, NUREG/CR-5813, EGG-2664, Vol. 1.

Russell, K. D., C. L. Atwood, M. B. Sattison, and D. M. Rasmuson, 1993, SAPHIRE Technical Reference Manual: IRRAS/SARA Version 4.0, NUREG/CR-5964, EGG-2692.

SAS Institute Inc. (1988), SAS/STAT User's Guide, Release 6.03 Edition, Cary, NC.

Siu, Nathan, and Ali Mosleh, 1989, "Treating Data Uncertainties in Common-Cause Failure Analysis," Nuclear Technology, Volume 84, pp. 265-280.

StatXact for SAS, 1994, Cambridge, MA: CYTEL Software Corp.

Walpole, Ronald E., and Raymond H. Myers, 1978, Probability and Statistics for Engineers and Scientists, New York: Macmillan. 
Appendix A

\section{SAS Programs}


A-2 


\section{Appendix A}

\section{SAS Programs}

This contains the SAS programs that were used to perform the analyses of this document.

\section{MACRO BCONFINT}

\%macro bconfint(datain, id, hits, trials, dotest);

$/ \star$

This program generates a $90 \%$ confidence interval for $p=$ Prob(hit), based on $k$ hits in $n$ trials, for each row in the data set.

To change the confidence level, change the values 0.05 and 0.95 in the calls to finv.

The method follows Johnson and Kotz, 1969, Discrete Distributions, Sec.3.7.2.

If requested, the macro also performs a contingency table test of the hypothesis that all rows in the data set have the same prob(hit). The test uses the approximate chi-square distribution. To request the exact test, change the word 'chisq' to 'exact' in the 'tables' option of proc freq. However, with large tables, this may cause SAS to take a very long time and then conclude that it cannot do the exact test.

In the explanation here, substitute your own word for anything in italics. Either type this macro early in the SAS batch file, or store it as filename and type \%include 'filename':

early in the SAS batch file.

After a data set has been defined, invoke the macro by the line \%bconfint (datain, var1, var2, var3, X)

Here datain is the name of the data set to be used (or last_ if the most recently referenced data set is to be used); var1, var2, and var3 are the names of variables in the data set corresponding to the record identifier, hits, and trials, respectively; and $X$ is the letter $T$ if the contingency-table test is to be carried out, and the letter $F$ otherwise. Do not put quotation marks around these arguments.

WARNING: This macro creates temporary data sets whose names begin with XXX. $\star 1$

The user should avoid such names, or accept that the macro may change them. 
DATA xxxbc1; set \&datain;

* calculate an extra record for the totals; PROC SUMMARY data $=x \times x b c 1$;

VAR \&hits \&trials;

OUTPUT OUT $=x \times x b c 2($ drop $=$ type__freq_) SUM=;

run:

DATA $x \times x b c 2$; set $x x \times b c 2$;

\&id $={ }^{\prime} \operatorname{Tota} T^{\prime}$;

call symput('exptprob', left(\&hits/\&trials));

run:

* add this record to the original data set;

DATA $x \times x b c 3$;

set $x \times x b c 1 \times x \times b c 2$ end=last;

a02 $=.05$ :

phat $=$ \&hits $/$ \&trials

$\mathrm{p} 10=0$

if (\&hits > 0) then do

nu170 $=2 *$ \&hits

nu210 $=2 *($ \&trials - \&hits +1$)$

nuFlo $=$ nu170*finv (ao2, nu110, nu270)

plo $=$ nuFlo $/($ nu210 + nuFlo $)$

end

pup $=1$

if (\&hits < \&trials) then do

nulup $=2^{*}($ \&hits +1$)$

nu2up $=2^{*}($ \&trials - \&hits $)$

nuFup $=$ nulup $\star$ finv $(1-$ ao2, nulup , nu2up $)$;

pup $=$ nuFup $/($ nu2up + nuFup $)$

end

if last then chisq $=$.;

else chisq=(\&hits-\&trials*\&exptprob)**2/(\&trials*\&exptprob*(1-\&exptprob));

run:

proc print noobs:

var \&id \&hits \&trials plo phat pup;

run:

\% if "\&dotest" = "T" \%then \%do;

DATA: set $x \times x b c 1$;

length outcome $\$ 12$.;

keep count outcome \&id :

count = \&hits

outcome = '\&hits' ;

output;

count $=$ \&trials - \&hits;

outcome = 'non-\&hits'

output:

run; 
proc freq:

weight count:

tables outcome*\&id / chisq nocol nopercent norow expected cellchi2 :

\%end;

run;

\%mend bconfint:

\section{MACRO BBAYES}

\%macro bbayes(datain, id, aprior, bprior, hits, trials);

/*

This macro finds a Bayes mean and $90 \%$ interval for binomial parameter, for each record of the data set. To change the content of the interval, change the arguments of betainv.

See comments for bconfint on how to include the macro in a SAS batch file. Below, substitute your own words for those in italics.

To invoke bbayes, after a data set has been created type \%bbayes(datain, var1, var2, var3, var4, var5)

Here, datain is the name of the data set to be used (or _last_ if the most recently referenced data set is to be used): varl is the name in the data set of the variable containing the record identifiers, var2 and var3 are either numbers or names in the data set for the parameters of the prior beta distribution, and var4 and var5 are names of variables in the data set corresponding to hits and trials.

*I

DATA; set \&datain;

$$
\begin{aligned}
& \text { apost }=\text { \&aprior }+ \text { \&hits } \\
& \text { bpost }=\text { \&bprior }+ \text { \&trials }- \text { \&hits } \\
& \text { plo }=\text { betainv }(.05, \text { apost }, \text { bpost }) \\
& \text { pmean = apost } / \text { (apost+bpost) } \\
& \text { pup = betainv }(.95, \text { apost } \text {. bpost })
\end{aligned}
$$

run;

proc print noobs:

var \&id apost bpost plo pmean pup;

run;

\%mend bbayes: 


\section{PROGRAM WMW}

/*

program WMW

This program was used to perform the Wilcoxon-Mann-Whitney test to see if Licensee Event Reports (LERS) reporting safety function loss (SFL) are uniformly distributed among the LERs, with the alternative being that they occur more often at one end of the sequence.

$\star 1$

title 'cumulative SFL LERs' :

DATA:

infile 'ordered.asc' ;

/*

The two relevant input variables were the date, in format month/day/year, and whether the event involved a loss of safety function, coded as a 1 for loss of safety function and a 0 otherwise.

*)

INPUT datchar MMDDYY8. fid $\$$ lernum $\$$ flmd $\$ s f l$

category = 'SFA':

if $(\mathrm{sf}\rceil=1)$ then category = 'SFL' ;

* Recode the dates so that a later date always is shown by a larger number ;

* The Julian date is one way. The format YYMMDD would work just as well; date $=$ juldate (datchar):

keep date category:

output:

run:

proc contents; run;

proc nparlway wilcoxon;

class category:

var date;

run; 
\%macro bbinmle(datain, dataout, fvar, dvar);

* Macro bbinmle

* Obtains MLE of parameters for beta-binomial distribution.

*

* First parameter $=$ input data set name

* Second parameter $=$ output data set name, containing parameter estimates

* Third parameter=failure variable name (e.g., hits)

* Fourth parameter=demand variable name (e.g., trials)

*

* Output data set contains variables aprior, bprior, varmu, vardel, covar,

* and dsum. These are a11 used by the macro bbempb:

* Currentiy dimensions are set for up to 150 records in the input data set.

* Curnent

data \&dataout; set \&datain end=eof nobs=nobs;

keep aprior bprior varmu vardel covar dsum;

array d[150]:

array f[150]:

retain $d f$ :

$\mathrm{d}\left[\mathrm{n}_{3}\right]=\& \mathrm{dvar}$;

$f[n]=\&$ fvar:

* Sum dvar and fvar:

dsum + \&dvar:

fsum + \&fvar:

if eof then do;

* find MLEs for mu and delta, and therefore for aprior and bprior;

muguess $=$ fsum $/$ dsum;

delhi = dsum;

de $110=0$

delta = delhi:

link dddelsub:

if (dddelLL $>0$ ) then do:

file print notitle:

put ................. TO LARGE-

put '...-Reverting to non-informative prior-.....';

file log;

aprior = fsum + 0.5:

bprior $=\mathrm{dsum}+0.5-$ fsum:

de 7 ta=aprior+bprior:

$\mathrm{mu}=\mathrm{aprior} / \mathrm{delta}$;

varmu $=0$;

varde $1=0$; 
covar $=0$

end:

else do:

* Bisection search for delta:

do while( abs(del10-delhi)>0.0005*delhi):

delta $=($ de $710+$ delhi $) / 2$;

link addelsub:

if dddelLL $>=0$ then de110 = delta;

if ddde $1 \mathrm{LL}<=0$ then delhi = delta;

end:

* MLEs found;

aprior $=$ delta*mu;

bprior $=\operatorname{delta*}(1-\mathrm{mu})$;

*Now find information matrix in terms of mu and delta;

sum11 $=0 ; \operatorname{sum} 22=0 ; \operatorname{sum} 33=0 ; \operatorname{sum} 12=0 ; \operatorname{sum} 21=0$;

do $i=1$ to nobs:

if $f[i]>0$ then do $j=0$ to $f[i]-1$;

sum11 $=$ sum $11+1 /($ mu*delta $+j) * \star 2 ;$

sum12 $=$ sum12 $+j /($ mu*delta $+j) \star \star 2 ;$

end:

if $f[i]<d[i]$ then do $j=0$ to $d[i]-f[i]-1$;

sum22 $=$ sum22 $+1 /((1-$ mu $) *$ delta $+j) * * 2 ;$

sum21 $=$ sum21 $+j /((1-m u) *$ delta $+j) * * 2$;

end:

do $j=0$ to $d[j]-1$ :

sum33 $=5$ um33 $+1 /($ detta $+j) * \star 2:$

end:

end;

infoll $=($ de 1 ta $* * 2) *(\operatorname{sum} 11+\operatorname{sum} 22)$;

info22 $=(m u * * 2) *$ sum11 $+((1-m u) * * 2) * \operatorname{sum} 22-\operatorname{sum} 33$;

info12 = sum21 - sum12;

det $=$ infol1*info $22-$ info $12 * \star 2$;

if det $<=0$ then do;

file print notitle:

put ' $\star \star \star \star \star E R R O R ! ! '$ infoll $1=$ info $22=$ infol $12=$ det $=$;

file log:

$\operatorname{det}=0$;

end;

varmu $=$ info22/det

varde $1=$ infoll/det

covar = - infol2/det;

sdvmu=sqrt (varmu):

sdvde $=$ sqrt $($ vardel $)$;

correl = covar/(sdvmu*sdvdel):

plo = betainv (.05, aprior,bprior):

pup = betainv (.95, aprior ,bprior):

file print: 
put:

put -Fitted Prior.............. '

putexpr="MLE Prior from "||left(fsum)||" failures and "I| left (dsum) |!" demands: "

put putexpr:

put aprior $=$ bprior $=$ plo $=$ mu $=$ pup $=$;

put $\mathrm{mu}=\mathrm{sdvmu}=$ varmu $=$;

put de 7 ta $=$ sdvde $]=$ varde $7=$;

put correl $=$ covar $=$;

put

file log:

end:

output;

end;

return:

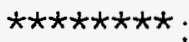

ddmusub:

/*

Calculate deriv wrt mu of loglikelihood, for fixed delta, and put value in ddmuLL

*1

sum1 $=0 ;$ sum2 $=0$;

do $i=1$ to nobs:

if $f[i]>0$ then do $j=0$ to $f[i]-1$;

sum1 $=$ sum $1+1 /\left(m u^{*} \operatorname{de} 1 t a+j\right) ;$

end:

if $f[i]<d[i]$ then do $j=0$ to $d[i]-f[i]-1$;

sum2 $=\operatorname{sum} 2+1 /((1-\mathrm{mu}) \star \operatorname{delta}+j)$ end:

end;

ddmuLL $=\operatorname{de} 7 t^{*}(\operatorname{sum} 1-\operatorname{sum} 2)$;

return;

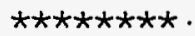

dddelsub:

/*

Calculate deriv wrt delta of loglikelihood, at mu that maximizes

loglikelihood for that delta.

We link ddmusub, and therefore use mu that makes ddmuLL $=0$.

Put value in dddel $L L$

$\star 1$.

* get MLE for mu, given delta:

* bracket mu:

mu = muguess:

Tink ddmusub:

if ddmuLL $<0$ then do whi $7 e(d d m u L L<0)$;

muhi = mu;

ddmuhi = ddmuLL;

mulo $=0.9 \star \mathrm{mu}$; 
mu $=$ mulo:

link ddmusub;

ddmulo = ddmuLL;

end:

else if ddmuLL $>0$ then do while (ddmuLL $>0$ );

mulo = mu:

ddmu $70=$ ddmuLL:

muhi $=1-0.9 \star(1-\mathrm{mu})$;

$\mathrm{mu}=$ muhi ;

Tink ddmusub:

ddmuhi = ddmuLL;

end:

else do:

mulo = mu:

muhi $=$ mu;

end:

* Now we have bracketed mu by mulo and muhi;

* Find mu by false position;

do while ( abs (mulo-muhi)>0.000001*muhi );

mu $=($ mulo*ddmuhi - muhi*ddmulo $) /($ ddmuhi - ddmulo $)$;

link ddmusub:

* PUT 'in dddelsub ' delta $=$ mu= ddmuLL= sum1 $=$ sum2 $=$;

if (ddmuLL > 0) then do;

mulo = mu;

ddmulo = ddmuLL:

/* Multiplication below is necessary in case function is strongly

* curved. We use .9 rather than a smaller number, because this

* particular function seems pretty smooth. */

ddmuhi $=.9^{*}$ ddmuhi ;

end:

else do:

muhi $=$ mu:

ddmuhi $=$ ddmuLL:

ddmulo $=.9{ }^{*}$ ddmulo:

end:

end;

* now calculate deriv of loglikelihood wrt delta;

ddde $1 \mathrm{LL}=0$;

sum3 $=0$;

do $j=1$ to nobs:

/*

Because we just linked ddmusub, sum2 has already been calculated

*)

do $j=0$ to $d[i]-1$

sum3 $=$ sum3 $+1 /($ delta $+j)$

end:

end;

ddde $1 L L=$ ddde $1 L L+($ sum2 - sum3):

* PUT ' in dddelsub' delta $=$ mu= sum $1=\operatorname{sum} 2=\operatorname{sum} 3=\operatorname{ddde} l \mathrm{~L} L=$; 
return;

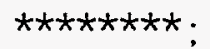

run;

\%mend bbinmle;

\section{MACRO BBEMPB}

\%macro bbempb(datain, dataemb, id, fvar, dvar);

/*

Finds Bayes posterior mean and 90\% interval for binomial parameter.

for each record.

Also finds whether any record is in tail of the prior distr.

datain = input data set with trial data. Typically, this is the same data set

that was used as an input to bbinmle.

dataemb = input data set containing empirical Bayes estimates. Typically,

this

is dataout from bbinmle. The variable names must aprior, bprior, varmu, vardel, and covar.

id = name of variable containing record identifier in datain

fvar = name of variable with failure counts (hits)

dvar = name of variable with demand counts (trials)

For example, if 'mydata' has variables 'plant', 'failures', and 'demands'. an empirical Bayes analysis by plant could be performed by the two 1 ines:

\%bbinmle (mydata, datatmp, failures, demands);

\%bbempb(mydata, datatmp, plant, failures, demands);

data; set \&datain NOBS=nobs end=last;

keep \&id apost bpost plo mupost pup adjapost adjbpost adjplo adjpup

leftprob rghtprob ;

retain delta mu ip logprobc:

if $\mathrm{N}_{-}=1$ then do;

set \&dataemb:

delta=aprior+bprior:

mu=aprior/delta;

$1 p=7$ gamma (delta) - Tgamma(aprior) - Tgamma(bprior);

logprobc=1gamma (delta) - 1gamma (bprior);

$\% *$ logprobc is the constant terms in logprob for 0 failures below:

end;

apost $=$ aprior $+\&$ fvar

bpost $=$ bprior $+\& d v a r-\& f v a r$ :

delpost $=$ delta + \&dvar:

mupost $=$ apost $/$ delpost:

plo $=\operatorname{betainv}(.05$, apost, bpost $)$;

pup $=\operatorname{betainv}(.95$, apost . bpost $)$ : 
* Next two Tines are (d mupost)/(d mu) and (d mupost)/(d delta) ; dmdmu $=$ delta/delpost :

dmddl $=($ \&dvar*mu $-\&$ fvar $) /($ delpost**2)

simplvar $=$ mupost $*(1$-mupost $) /($ delpost +1$)$;

* This is the Kass-Steffey adjusted variance;

adjvar $=$ simplvar $+($ dmdmu**2)*varmu $+($ dmdd $7 * * 2) *$ varde 1

$+2 * d m d m u * d m d d 1 *$ covar ;

adjdpost $=($ mupost $*(1$-mupost $) /$ adjvar $)-1$;

adjapost $=$ mupost*adjdpost :

adjbpost $=(1-\text { mupost })^{\star}$ adjdpost

adjplo $=\operatorname{betainv}(.05$, adjapost, adjbpost $)$;

adjpup $=\operatorname{betainv}(.95$, adjapost, adjbpost $):$

* Find tail prob. ( $p$-vaTue) of data, under beta-binomial assump.;

if $(\&$ fvar $=0)$ then do :

rghtprob $=1$;

logprob = logprobc + 1gamma (bprior + \&dvar) - lgamma(delpost) ;

leftprob $=\exp ($ logprob $)$ : end:

else do :

$1 p p=7 p-7 g a m m a(d e$ post $)+7 g a m m a(\& d v a r+1) ;$

rghtprob $=0$;

do $j=\& d v a r$ to \&fvar by -1 ;

probi $=\exp (7 p p+7 g a m m a(a p r i o r+i)$

+ Tgamma (bprior + \&dvar - i)

- Igamma $(i+1)-\operatorname{lgamma}(\& d v a r-i+1))$;

rghtprob $=$ rghtprob + probi $;$

end;

leftprob $=1-$ rghtprob + probi ;

end;

run;

proc print noobs; run;

\%mend bbempb; 
Appendix B

Technical Details 
B-2 


\section{Appendix B}

\section{Technical Details}

\section{CONFIDENCE INTERVALS AND BAYES INTERVALS}

This section discusses the relation between confidence intervals and Bayes intervals for a binomial parameter $p$, based on $k$ hits in $n$ trials. Philosophically, there is no relation at all. The frequentist approach, leading to confidence intervals, treats $p$ as fixed and the data value $k$ as random, while the Bayes approach treats $p$ as random and the data value $k$ (after it has been observed) as fixed. Nevertheless, reasonable people have adopted both approaches and obtained credible answers. Therefore, it is not too surprising that there is a numerical relation between confidence intervals and Bayes intervals. This section makes this numerical comparison, and finds that the two kinds of intervals are inherently similar numerically when the Bayes method uses a noninformative prior.

True Bayesians may feel no need to see the relation between Bayes intervals and confidence intervals. Frequentists, on the other hand, prefer confidence intervals, but may be forced to use Bayes distributions because the standard methods of PRA are Bayesian. The comparisons here may therefore help frequentists to find Bayesian methods palatable, at least when noninformative prior distributions are used.

For simplicity of presentation, the desired confidence level is always assumed to be $90 \%$, corresponding to tail probabilities of 0.05 . The subscripts 'conf' and 'Bayes' will be used to distinguish between confidence limits and Bayes limits.

\section{Confidence Intervals}

First, exact $90 \%$ confidence cannot be achieved, because the binomial random variable is discrete. The desired statement at the lower end is that

$P\left(p \leq p_{\text {conf.L }}\right)=0.05$

for all $p$. Here $p_{\text {conf, } \mathrm{L}}$ is the lower end of the confidence interval, calculated from the random data. The number of hits in $n$ trials can only take $n+1$ values, from 0 to $n$. Therefore, no matter how cleverly we 
adjust the formula for the lower limit $p_{\text {conf,L }}$, it can only take a finite number of values, and it is therefore not possible to make Equation (B-1) exactly true for all $p$. Instead, it is customary to adopt a conservative definition, and require that

$P\left(p \leq p_{\text {conf }, \mathrm{L}}\right) \leq 0.05$

and similarly for the upper point we require that

$P\left(p \geq p_{\text {conf, } \mathrm{U}}\right) \leq 0.05$

This leads to an interval with at least $90 \%$ confidence of covering the true value of $p$.

The confidence interval can be constructed in principle by recalling that the confidence interval consists of all values $p_{0}$ that would not be rejected by a test of the hypothesis $H_{0}: p=p_{0}$. For the lower endpoint, the appropriate test is a one-sided test of $H_{0}$ versus $H_{1}: p>p_{0} . H_{0}$ is rejected, based on $k$ observed hits in $n$ trials if

$P\left(k\right.$ or more hits $\left.\mid p_{0}\right) \leq \alpha / 2$

that is

$\sum_{i=k}^{n}\left[\begin{array}{c}n \\ i\end{array}\right] p_{0}{ }^{i}\left(1-p_{0}\right)^{n-i} \leq \alpha / 2$

and the confidence limit $p_{\text {conf,L }}$ is the largest of the $p_{0}$ values, that is, the value for which equality is achieved. The sum can be shown to equal the integral of the beta $(k, n-k+1)$ density from 0 to $p_{0}$. This is the integral that was written $I\left(p_{0} ; k, n-k+1\right)$ in the body of the report. To prove that the sum and the beta integral are equal, note that the integral is

$\frac{n !}{(k-1) !(n-k) !} \int_{0}^{p_{0}} t^{k}(1-t)^{(n-k)} d t$,

and integrate it by parts $n-k$ times. This derives Equation (2a) in the body of this report, and Equation (2b) is derived similarly. They are repeated here as (B-2a) and (B-2b): 
$I\left(p_{\text {conf }, L} ; k, n-k+1\right)=\alpha / 2$

$I\left(p_{\text {conf }, U} ; k+1, n-k\right)=1-\alpha / 2$

Note, the parameters are slightly different in the two equations.

The reexpression of a beta integral in terms of the $F$ distribution is based on the fact that if $Y$ has an $F\left(\nu_{1}, \nu_{2}\right)$ distribution, then $\nu_{1} Y /\left(\nu_{2}+\nu_{1} Y\right)$ has a beta $\left(\nu_{1} / 2, \nu_{2} / 2\right)$ distribution. This is shown by letting $X_{1}$ and $X_{2}$ be independent $\chi^{2}$ random variables with $\nu_{1}$ and $\nu_{2}$ degrees of freedom. Then $Y \equiv\left(X_{1} / \nu_{1}\right) /$ $\left(X_{2} / \nu_{2}\right)$ has the $F$ distribution (Johnson and Kotz, 1970, Section 26.1) and $Z \equiv X_{1} /\left(X_{1}+X_{2}\right)$ has the beta distribution (Johnson and Kotz, 1970, Section 24.2). Some algebra yields $Z=\nu_{1} Y /\left(\nu_{2}+\nu_{1} Y\right.$ ), which is the asserted relation between the $F$ and beta random variables, Equation (3) of the body of this report.

Therefore, as presented in the body of this report, the confidence limits are

$p_{\text {conf }, \mathrm{L}}=\nu_{1} F_{0.05} /\left(\nu_{2}+\nu_{1} F_{0.05}\right) \quad$ with $\nu_{1}=2 k, \nu_{2}=2(n-k+1)$,

$p_{\text {conf }, \mathrm{U}}=\nu_{1} F_{0.95} /\left(\nu_{2}+\nu_{1} F_{0.95}\right) \quad$ with $\nu_{1}=2(k+1), \nu_{2}=2(n-k)$

where $F_{q}$ is the $q$ th quantile of the $F$ distribution with $\nu_{1}$ and $\nu_{2}$ degrees of freedom.

Suppose instead that we wanted an interval with at most $90 \%$ confidence. This is not normally done, but it will be instructive as we compare confidence intervals and Bayes intervals. We would use the above formulas, but for the lower end act as if there had been one more hit than observed, and for the upper end act as if there had been one fewer hit than observed. The result is to adjust the parameters of the beta distribution, or the corresponding degrees of freedom of the $F$ distribution:

$I\left(p_{\text {conf,L }} ; k+1, n-k\right)=\alpha / 2$

$I\left(p_{\text {conf }, \mathrm{U}} ; k, n-k+1\right)=1-\alpha / 2$

These formulas will be recalled in the following discussion of Bayes intervals.

\section{Bayes Intervals}

Now consider the Bayesian interval, with $p$ treated as a random variable. We will derive equations that are very similar to Equations (B-2) and (B-3), only the degrees of freedom will be different; this will allow a direct comparison of the Bayes interval and the confidence interval. 
Based on the noninformative prior, the posterior distribution for $p$ is beta $(k+1 / 2, n-k+1 / 2)$. As mentioned in the body of this report, this results in endpoints satisfying

$I\left(p_{\text {Bayes, },} ; k+1 / 2, n-k+1 / 2\right)=\alpha / 2$

$I\left(p_{\text {Bayes, },} ; k+1 / 2, n-k+1 / 2\right)=1-\alpha / 2$

Note, the parameters are the same for both ends of the interval, because they correspond to the single posterior distribution. These equations are numerically the same as the (B-2) and (B-3), except the parameters average the parameters from (B-2) and (B-3).

Therefore, the Bayes interval may be thought of, numerically, as a compromise between Equations (B-2) and (B-3), that is, a compromise between having at least $90 \%$ confidence and at most $90 \%$ confidence. Numerically, it is an approximate correction for the discreteness of the data. 


\section{EMPIRICAL BAYES METHOD}

\section{Beta-Binomial Likelihood}

If the number of hits in $n$ trials, given $p$, has a binomial $(n, p)$ distribution, and if $p$ has a beta( $\alpha$, $\beta$ ) distribution, then the unconditional distribution of the number of hits is called beta-binomial $(n, \alpha, \beta)$. It is a straightforward exercise using formulas involving the beta function and the gamma function to show that the probability function of the beta-binomial distribution is

$$
\begin{aligned}
P(k) & =\frac{n !}{k !(n-k) !} \frac{\Gamma(\alpha+k)}{\Gamma(\alpha)} \frac{\Gamma(\beta+n-k)}{\Gamma(\beta)} \frac{\Gamma(\alpha+\beta)}{\Gamma(\alpha+\beta+n)} \\
& =\frac{n !}{k !(n-k) !} \prod_{j=0}^{k-1}(\alpha+j) \prod_{j=0}^{n-k-1}(\beta+j) / \prod_{j=0}^{n-1}(\alpha+\beta+j)
\end{aligned}
$$

where the second line follows from the first by repeated use of the relation $\Gamma(a)=(a-1) \Gamma(a-1)$ for any $a>1$. When there are a number of plants, with the $i$ th plant having $k_{i}$ hits in $n_{i}$ trials, the $\log$-likelihood is therefore

$L=\sum_{i}\left[\sum_{j=0}^{k_{i}-1} \ln (\alpha+j)+\sum_{j=0}^{n_{i}-k_{i}-1} \ln (\beta+j)-\sum_{j=0}^{n_{i}-1} \ln (\alpha+\beta+j)\right]+K$

where $K$ does not depend on $\alpha$ or $\beta$. Many computer packages, including SAS, have $\Gamma, \ln \Gamma$, and the derivative of $\ln \Gamma$ as built-in functions. Nevertheless, with SAS we were more successful in maximizing the $\log$-likelihood when we expressed it using Equation (B-5), which does not use the $\Gamma$ function.

\section{Maximum Likelihood Estimation}

Maximization in other programming languages may be easier, but we use SAS for consistency with the rest of this report. The algorithm used by the SAS macro bbinmle is derived as follows.

To obtain the maximum likelihood estimates $\hat{\alpha}$ and $\hat{\beta}$, the log-likelihood must be maximized numerically. For this, it is advantageous to reparameterize in terms of $\delta=\alpha+\beta$ and $\mu=\alpha / \delta$. The intuitive reason is that in most models the mean is one of the easiest things to estimate. Therefore we parameterize the model in terms of the mean $\mu$, and another quantity that is not directly related to the mean. For the present model, $\delta$ seems natural, as it is related to the variance of a beta distribution: var $=\mu(1-\mu) /(\delta+1)$. We will refer to $\delta$ as the dispersion parameter; the letter $\delta$ was chosen as a mnemonic 
for "dispersion." Some three-dimensional plots of the log-likelihood with example data show a ridge roughly parallel to the $\mu$ axis and roughly perpendicular to the $\delta$ axis, with the surface changing rapidly with $\mu$ and slowly with $\delta$.

Write the $\log$-likelihood in terms of $\mu$ and $\delta$, by substituting $\mu \delta$ for $\alpha$ and $(1-\mu) \delta$ for $\beta$ in Expression (B-5), obtaining

$L=\sum_{i}\left[\sum_{j=0}^{k_{i}-1} \ln (\mu \delta+j)+\sum_{j=0}^{n_{i}-k_{i}-1} \ln [(1-\mu) \delta+j]-\sum_{j=0}^{n_{i}-1} \ln (\delta+j)\right]+K$

where $K$ does not depend on $\mu$ or $\delta$. Denote the $\log$-likelihood, $L$, by $L(\mu, \delta)$ if the parametric dependence must be shown. For $\delta$ fixed, the first derivative with respect to $\mu$ is

$$
\frac{\partial L}{\partial \mu}=\sum_{i}\left[\sum_{j=0}^{k_{i}-1} \frac{\delta}{\mu \delta+j}-\sum_{j=0}^{n_{i}-k_{i}-1} \frac{\delta}{(1-\mu) \delta+j}\right]
$$

At the MLE, this derivative equals 0 . The second partial derivative $\partial^{2} L / \partial \mu^{2}$ can be seen to be always negative, so the first derivative $\partial L / \partial \mu$ is monotone decreasing. Define $\mu(\delta)$ as the value that makes Expression (B-7) equal to zero. After a small number of calculations to bracket $\mu(\delta)$, the method of false position typically needs six or fewer iterations to find $\mu(\delta)$ to five- or six-digit accuracy. Accuracy is required, because the log-likelihood is sensitive to the value of $\mu$, and we now must use this value in the derivative with respect to $\delta$, whose calculation involves subtraction of two nearly equal numbers.

Symbolically substitute $\mu(\delta)$ into $L(\mu, \delta)$. By elementary formulas of multivariate calculus, the derivative of $L(\mu(\delta), \delta)$ is

$$
\begin{aligned}
\frac{d L(\mu(\delta), \delta)}{d \delta} & =\frac{\partial L(\mu, \delta)}{\partial \mu} \frac{d \mu(\delta)}{d \delta}+\frac{\partial L(\mu, \delta)}{\partial \delta} \text {, evaluated at } \mu=\mu(\delta) \\
& =0 \times \frac{d \mu}{d \delta}+\frac{\partial L(\mu, \delta)}{\partial \delta}, \text { evaluated at } \mu=\mu(\delta) \\
& =\frac{\partial L(\mu, \delta)}{\partial \delta}, \text { evaluated at } \mu=\mu(\delta)
\end{aligned}
$$

where the zero term in the second line follows from the definition of $\mu(\delta)$. This derivative is

$$
\left.\frac{\partial L}{\partial \delta}\right|_{\mu=\mu(\delta)}=\sum_{i}\left[\sum_{j=0}^{n_{i}-k_{i}-1} \frac{1}{(1-\mu) \delta+j}-\sum_{j=0}^{n_{i}-1} \frac{1}{\delta+j}\right]
$$

The derivative of the log-likelihood has more terms, but at $\mu(\delta)$ some of those terms sum to zero, and therefore do not appear in Equation (B-8). 
If this derivative is positive when $\delta$ equals the total number of trials in the data set (temporarily denoted $n$ here), the algorithm stops. Either the maximization procedure does not converge, or it yields a $\delta>n$, which is unreasonable, as discussed in the next paragraph. On the other hand, if the derivative is negative when $\delta=n$, the algorithm finds a value of $\delta$ between 0 and $n$ where the derivative is zero. The bisection method typically requires about fifteen iterations to achieve three-digit accuracy; the method of false position, though more sophisticated, did not improve the speed of convergence in two test problems, apparently because it relied too much on exact calculation of the derivatives. This numerical search yields the MLEs, $\hat{\delta}$ and $\hat{\mu}=\mu(\hat{\delta})$. The second derivatives of $L$ are then found at the MLEs, to be used in the Kass-Steffey adjustment described below.

To see why $\delta>n$ is unreasonable, note that the posterior distribution for the $i$ th plant is a beta distribution with dispersion parameter $\delta_{i}=\delta+n_{i}$. Now suppose that instead of using the empirical Bayes method, we simply pooled the data and used the noninformative prior distribution. The distribution for $p$ would be beta with dispersion parameter $\delta_{\text {post }}=n+1$. If $\delta$ is greater than $n$, then $\delta_{i}$ is larger than $\delta_{\text {post }}$ - This says that, except for the effect of different means, the empirical Bayes posterior distribution for the $i$ th plant has a smaller variance than the posterior distribution based on pooling all the data. This is unreasonable.

\section{Adjustment for Uncertainty in Maximum Likelihood Estimates}

Kass and Steffey (1989) present an adjustment to account for the fact that the parameters $\alpha$ and $\beta$ (or equivalently $\mu$ and $\delta$ ) are only estimated from data, not known exactly. This method is applied to the beta-binomial model by Martz, Abramson, and Kvam (1994). The method is based on moments, and involves the variances and covariance of the parameter estimators. Kass and Steffey state that the adjustment is very important if there are few data sources (such as plants) and many observations (trials), and conversely that the adjustment is unimportant when there are many data sources and few observations. The SAS macros bbinmle and bbempb make the adjustment automatically, because it is computationally quick and there is never any harm in doing it.

We must consider which parameterization to use. For maximum likelihood estimators, the variancecovariance matrix is estimated by inverting the sample information matrix, that is, the negative of the matrix of second derivatives of the log-likelihood. In example problems, if this matrix is constructed by differentiating with respect to $\alpha$ and $\beta$, the matrix is more nearly singular (corresponding to a correlation of 0.8 or 0.9 ) than if the derivatives are with respect to $\mu$ and $\delta$ (corresponding to a correlation of about 0.2 ). The information matrix is evaluated at the parameter estimates. Therefore, any error in the 
estimates will affect the calculated inverse much more if the matrix is nearly singular than if the matrix is nearly diagonal. This difference in parameterization was very important in a study by Atwood (1984); although it may not be so important here, we have to choose one parameterization, and we choose to use $\mu$ and $\delta$. Sometimes below we will write $\alpha$ as shorthand for $\mu \delta$, but $\mu$ and $\delta$ are still the fundamental parameters.

Let $p_{i}$ denote the probability of a hit at the $i$ th plant. We wish to find the posterior mean and variance of $p_{i}$ :

$E\left(p_{i} \mid\right.$ all the data $)$

$\operatorname{Var}\left(p_{i} \mid\right.$ all the data $)$

The simple empirical Bayes method approximates these by

$E\left(p_{i} \mid k_{i}, n_{i}, \hat{\mu}, \hat{\delta}\right)=\left(\hat{\alpha}+k_{i}\right) /\left(\hat{\delta}+n_{i}\right)$, denoted $\hat{\mu}_{i}$

$\left.\operatorname{Var}\left(p_{i} \mid k_{i}, n_{i}, \hat{\mu}, \hat{\delta}\right)=\hat{\mu}_{i}\left(1-\hat{\mu}_{i}\right) / \hat{\delta}+n_{i}\right)$

The Kass-Steffey adjustment estimates the posterior mean for the $i$ th data source by $\hat{\mu}_{i}$, but it increases the variance estimate to

$\operatorname{Var}\left(p_{i} \mid k_{i}, n_{i}, \mu, \delta\right)+\left[\frac{\partial \mu_{i}}{\partial \mu}\right]^{2} \operatorname{Var}(\hat{\mu})+\left[\frac{\partial \mu_{i}}{\partial \delta}\right]^{2} \operatorname{Var}(\hat{\delta})+2\left[\frac{\partial \mu_{i}}{\partial \mu}\right]\left[\frac{\partial \mu_{i}}{\partial \delta}\right] \operatorname{Cov}(\hat{\mu}, \hat{\delta})$

where

$\mu_{i}=\left(\alpha+k_{i}\right) /\left(\delta+n_{i}\right)=\left(\mu \delta+k_{i}\right) /\left(\delta+n_{i}\right)$. The variance and covariance terms are estimated by evaluating the information matrix

$$
-\left[\begin{array}{cc}
\frac{\partial^{2} L}{\partial \mu^{2}} & \frac{\partial^{2} L}{\partial \mu \partial \delta} \\
\frac{\partial^{2} L}{\partial \mu \partial \delta} & \frac{\partial^{2} L}{\partial \delta^{2}}
\end{array}\right]
$$


evaluated at $\hat{\mu}$ and $\hat{\delta}$. The inverse of this matrix is the estimated variance-covariance matrix of $(\hat{\mu}, \hat{\delta})$. These estimated variances are found by the SAS macro bbinmle.

The Kass-Steffey adjustment concludes by finding the values of $\alpha$ and $\beta$ that correspond to the estimated posterior mean and variance. These values are used to define the posterior distribution of $p_{i}$, modeled as a beta distribution. The SAS macro bbempb performs these calculations, and finds the posterior $90 \%$ interval for each $p_{i}$.

It might be objected that the asymptotic distribution for $(\hat{\mu}, \hat{\delta})$ is not very accurate. In particular, $\hat{\mu}$ appears all right, but in example data sets the calculated standard deviation of $\hat{\delta}$ is about the same size as $\hat{\delta}$. This indicates that the distribution of $\hat{\delta}$ is strongly skewed, and not close to the asymptotically normal distribution. This motivates use of a different parameter, such as log $\delta$. When the entire KassSteffey procedure is carried out with $(\mu, \log \delta)$ instead of $(\mu, \delta)$, the derivative and variance terms are all divided by $\delta$ or $\delta^{2}$, the $\delta$ 's cancel everywhere, and exactly the same formulas are obtained as above. Therefore, the formulas are used.

\section{Moment Estimation}

A numerically simpler method than maximum likelihood estimation is to match moments. The moment estimators $\alpha^{*}$ and $\beta^{*}$ are found by Martz and Waller (1991) as follows. (Warning: This reference has been printed by two publishers, and some of the formulas are incorrect in the first printing of the Wiley edition.) Define

$n=\Sigma n_{i}, \quad k=\Sigma k_{i}, \quad w=\Sigma\left(k_{i}^{2} / n_{i}\right) / n, \quad N=$ the number of plants.

Then the moment estimators of $\mu$ and $\delta$ are $\mu^{*}=k / n$ and

$\delta^{*}=n\left(\mu^{*}-w\right) /\left[n w-N \mu^{*}-(n-N)\left(\mu^{*}\right)^{2}\right]$.

The corresponding estimators of $\alpha$ and $\beta$ are $\alpha^{*}=\mu^{*} \delta^{*}$ and $\beta^{*}=\left(1-\mu^{*}\right) \delta^{*}$. These estimates are not necessarily positive, but they can be found directly, without numerical iteration. For that matter, the maximum likelihood estimates are not always positive and finite. However, in limited experience with various data sets, it seems that the maximum likelihood estimates are finite and positive more frequently than the moment estimates are. 


\section{REFERENCES}

Atwood, Corwin L., 1984, “Approximate Tolerance Intervals, Based on Maximum Likelihood Estimates," Technometrics, 79, pp. 459-465.

Johnson, Norman L., and Samuel Kotz, 1970, Continuous Univariate Distributions-2, New York: John Wiley \& Sons.

Kass, Robert E. and Duane Steffey, 1989, “Approximate Bayesian Inference in Conditionally Independent Hierarchical Models (Parametric Empirical Bayes Models)," Journal of the American Statistical Association, 84, pp. 717-726.

Martz, Harry F., Lee R. Abramson, and Paul H. Kvam, 1984, "Empirical Bayes Estimation of the Reliability of Nuclear Power Plant Emergency Diesel Generators: An Expository Application," LA-UR-93-1239 Rev. 2 (submitted to Technometrics).

Martz, Harry F., and Ray A. Waller, 1991, Bayesian Reliability Analysis, Malabar, FL: Krieger, Section 7.6 . 